\title{
MODULAR GRAPH FUNCTIONS
}

\author{
ERIC D'HOKER, MICHAEL B. GREEN, ÖMER GÜRDOĞAN, AND PIERRE VANHOVE
}

\begin{abstract}
In earlier work we studied features of non-holomorphic modular functions associated with Feynman graphs for a conformal scalar field theory on a twodimensional torus with zero external momenta at all vertices. Such functions, which we will refer to as modular graph functions, arise, for example, in the low energy expansion of genus-one Type II superstring amplitudes. We here introduce a class of single-valued elliptic multiple polylogarithms, which are defined as elliptic functions associated with Feynman graphs with vanishing external momenta at all but two vertices. These functions depend on a coordinate, $\zeta$, on the elliptic curve and reduce to modular graph functions when $\zeta$ is set equal to 1 . We demonstrate that these single-valued elliptic multiple polylogarithms are linear combinations of multiple polylogarithms, and that modular graph functions are sums of single-valued elliptic multiple polylogarithms evaluated at the identity of the elliptic curve, in both cases with rational coefficients. This insight suggests the many interrelations between modular graph functions (a few of which were established in earlier papers) may be obtained as a consequence of identities involving multiple polylogarithms, and explains an earlier observation that the coefficients of the Laurent polynomial at the cusp are given by rational numbers times single-valued multiple zeta values.
\end{abstract}

\section{Contents}

1. Introduction

2. Some basic features of multiple polylogarithms

3. Feynman graphs associated with a torus

4. Examples of modular graph functions 17

5. Single-valued multiple polylogarithms for graphs 20

6. Fourier coefficients of modular graph functions 28

7. Summary and thoughts on the basis of modular graph functions 32

Acknowledgments

Appendix A. Polylogarithms and Eichler integrals 34

Appendix B. The $C_{1,1,1}(q)$ modular graph function 35

Appendix C. Reduction of multiple sums to multiple polylogarithms 39

References

Date: December 14, 2016.

DAMTP-2015-86, IPhT-t15/202, IHES/P/15/29, LPTENS-15/09. 


\section{INTRODUCTION}

Superstring perturbation theory may be merely an approximation to a complete non-perturbative formulation of string theory, but it already exhibits a remarkably rich mathematical structure. The perturbative series is given by a topological expansion for two-dimensional surfaces which represent string world-sheets. For the closed superstring theories the perturbative series is given by the sum over all genera $g$, with $g \geq 0$, of functional integrals over orientable (super)Riemann surfaces. In the case of four-graviton amplitudes, which will be the prototype and point of departure of this paper, the explicit expressions for tree-level $(g=0)$ and genus-one $(g=1)$ were obtained in [1], for genus-two $(g=2)$ in [2], and a certain amount is known about the leading low energy behaviour of the genus-three $(g=3)$ case in [3]. For the open string theory, a summation over boundaries and cross-caps must also be included.

The low energy expansion of string theory corresponds to an expansion valid when the energies and momenta are small in units of the inverse of the string length scale $\ell_{s}$, a parameter which is related to Newton's gravitational constant. The lowest order contribution corresponds to Einstein's theory, while higher order corrections become important for strong gravitational fields. The structure of these higher order corrections is of considerable mathematical interest. In particular, their coefficients in the low energy expansion of tree-level $N$-particle amplitudes in open superstring theory are multiple zeta values, which are special values of multiple polylogarithms. The analogous coefficients of tree-level $N$-particle closed superstring amplitudes are singlevalued multiple zetas $[4,5]$, which in turn are special values of single-valued multiple polylogarithms, following the terminology introduced in [6-8].

Much less is understood about the low energy expansion of string amplitudes with higher genus. In the genus-one closed superstring case the coefficients in the low energy expansion are given by integrals of non-holomorphic modular functions over the complex structure modulus $\tau$ of the torus that is defined by the string world-sheet. These modular functions, which can be expressed as multiple sums, are generalisations of non-holomorphic Eisenstein series. Specific classes of such functions have been shown to satisfy a number of very intriguing relationships [9-11]. These are reminiscent of the algebraic relationships between multiple zeta values but, in the present case they are relationships between functions defined on an elliptic curve. In the genus-two closed superstring case, a connection has been uncovered with the Zhang-Kawazumi invariant [12], which satisfies equally intriguing relations [13], but whose study remains incomplete.

In the present paper, we specialise to the case of a conformal scalar field theory on a two-dimensional torus, or elliptic curve, with arbitrary complex modulus $\tau$. To every Feynman graph with vanishing external momenta on each vertex, $L$ loops, and $w$ scalar Green functions on the edges of the graph, we associate a non-holomorphic modular function in $\tau$ of depth $L$ and weight $w$, which we shall refer to as a modular 
graph function. Certain classes of graphs will produce vanishing modular functions, such as any one-edge reducible graph, and any graph in which at least one vertex supports only a single Green function edge. In this paper, we shall consider graphs with non-derivative couplings only, but this restriction can be easily lifted if needed.

We shall also introduce elliptic functions that depend on a point $\zeta$ on the elliptic curve of modulus $\tau$, and that are associated with Feynman graphs in which all but two vertices have vanishing external momenta. These elliptic functions provide examples of single-valued elliptic multiple polylogarithms. We will demonstrate that any modular graph function may be expressed as the value of a single-valued elliptic multiple polylogarithm ${ }^{1}$ when $\zeta$ is set equal to the particular value $\zeta=1$ corresponding to the identity on the elliptic curve. This is an elliptic analogue of the familiar statement that the single-valued multiple-zeta values discussed in [6-8] are the values of single-valued multiple polylogarithms with their arguments set equal to 1. It is therefore natural to call these special values single-valued elliptic multiple zetas. This connection between modular graph functions and single-valued elliptic multiple polylogarithms suggests a compelling origin of the many interrelations between modular graph functions (a few of which were motivated in [9]) as a consequence of identities involving elliptic polylogarithms.

1.1. Outline of paper. Section 2 will give a brief overview of some of the relevant features of polylogarithms, multiple polylogarithms, multiple zeta values, their singlevalued projections, and their elliptic generalisations that will enter into the subsequent ideas in the paper. Section 3 will discuss the modular graph functions and singlevalued elliptic multiple polylogarithms that arise in the low energy expansion of the perturbative amplitudes in superstring theory and which are expressed in terms of Feynman graphs for a conformal scalar field theory on a two-dimensional torus. In particular, we will show that every modular graph function is given by a single-valued elliptic multiple polylogarithm evaluated at a special point. Section 4 illustrates this feature by considering some of the infinite classes of graphs studied in $[9-11,17,18]$ by other methods. Section 5 develops the conjecture which states that single-valued multiple polylogarithms are linear combinations of elliptic polylogarithms with rational coefficients, offers a proof for the infinite class of star graphs, and outlines some of the arguments for general graphs. In section 6 , the validity of the conjecture is shown to lead to a corollary stating that the non-leading coefficients of the Laurent expansion of the constant Fourier mode of modular graph functions are single-valued multiple zeta values. A summary and further thoughts on the basis of modular graph functions, and their further generalisations, is relayed to section 7 .

\footnotetext{
${ }^{1}$ The elliptic multiple polylogarithms considered in this paper are not holomorphic and differ from those introduced in [14], which are of relevance to the open string annulus amplitude, as discussed in $[15,16]$. Although there is a clear relationship between the open string and the closed string, this relationship is not a subject studied in this paper.
} 
Appendix A presents some relations between Eichler integrals, elliptic polylogarithms, and holomorhic Eisenstein series in preparation of appendix B where the algebraic properties of multiple polylogarithms will be used to evaluate the modular graph function associated with the simplest two-loop graph, $C_{1,1,1}(q)$. This calculation makes use of various reduction identities for multiple sums that are determined in appendix $\mathrm{C}$.

\section{Some Basic features of multiple polylogarithms}

The emphasis in this paper is the analogy between elliptic functions that arise as coefficients in the low energy expansion in genus-one closed superstring amplitudes with single-valued multi-zetas that arise in the expansion of the tree-level amplitudes [4,5]. For clarity we will here give a brief (and incomplete) review of the relationship of these quantities to multiple polylogarithms and to single-valued multiple polylogarithms. There are many detailed reviews of this large subject in the literature and we note in particular the elementary introduction in [19].

2.1. Polylogarithms. The polylogarithm $\operatorname{Li}_{a}(z)$ is defined for any value of $a \in \mathbb{C}$ by the power series expansion

$$
\operatorname{Li}_{a}(z)=\sum_{k=1}^{\infty} \frac{z^{k}}{k^{a}}
$$

which is absolutely convergent for $|z|<1$. The polylogarithm is a natural generalisation of the $\operatorname{logarithm}$ since we have $\operatorname{Li}_{1}(z)=-\log (1-z)$. Alternatively, the function $\mathrm{Li}_{a}(z)$ may be defined by the integral representation

$$
\operatorname{Li}_{a}(z)=\frac{z}{\Gamma(a)} \int_{1}^{\infty} \frac{d t}{t} \frac{(\log t)^{a-1}}{t-z}
$$

which coincides with (2.1) for $|z|<1$, but may be analytically continued to all $z \in$ $\mathbb{C} \backslash\left[1,+\infty\right.$. The resulting $\operatorname{Li}_{a}(z)$ manifestly has a branch point at $z=1$, but it also has a branch point at $z=0$ on its higher Riemann sheets. Therefore, the function $\operatorname{Li}_{a}(z)$ for generic values $a \in \mathbb{C}$ is multiple-valued and has interesting monodromies, again generalising the properties of $\operatorname{Li}_{1}(z)=-\ln (1-z)$. Of particular interest is the relation of $\mathrm{Li}_{a}(z)$ to the Riemann zeta function $\zeta(a)$ via

$$
\operatorname{Li}_{a}(1)=\zeta(a),
$$

and to the Bernoulli polynomials $B_{n}(x)$ via

$$
\operatorname{Li}_{n}\left(e^{2 i \pi x}\right)+(-1)^{n} \operatorname{Li}_{n}\left(e^{-2 i \pi x}\right)=-\frac{(2 i \pi)^{n}}{n !} B_{n}(x),
$$


for $n \in \mathbb{N}$ and $x \in[0,1]$. Recall that $B_{n}(x)$ is the $n$-th Bernoulli polynomial which is defined by the expansion in powers of $t$ of the following generating function

$$
\frac{t e^{x t}}{e^{t}-1}=\sum_{n=0}^{\infty} B_{n}(x) \frac{t^{n}}{n !} .
$$

Moreover, the function $\operatorname{Li}_{a}(z)$ satisfies the differential relation

$$
z \frac{\partial \mathrm{Li}_{a}(z)}{\partial z}=\mathrm{Li}_{a-1}(z)
$$

and the converse integral relation

$$
\operatorname{Li}_{a+1}(z)=\int_{0}^{z} \frac{d y}{y} \operatorname{Li}_{a}(y) .
$$

2.2. Single-valued polylogarithms. At the cost of giving up holomorphicity in $z$, it is possible to construct a single-valued polylogarithm associated with $\operatorname{Li}_{a}(z)$. The Bloch-Wigner dilogarithm is the prototype for such single-valued polylogarithms, and is defined as follows

$$
D(z)=\Im \mathrm{m}\left(\operatorname{Li}_{2}(z)+\log (1-z) \log |z|\right), \quad z \in \mathbb{C} \backslash\{0,1\} .
$$

In this paper we will make use of the generalisations of the Bloch-Wigner dilogarithm introduced by Zagier in [20]. They are single-valued (real analytic) polylogarithms $D_{a, b}(z)$ for $z \in \mathbb{C} \backslash[1, \infty[$ and $a, b \in \mathbb{N}$, and can be expressed in terms of sums of ordinary polylogarithms $\operatorname{Li}_{k}(z)$ for an integer index $k$ by

$$
\begin{aligned}
D_{a, b}(z)=(-1)^{a-1} \sum_{k=a}^{a+b-1} & \left(\begin{array}{c}
k-1 \\
a-1
\end{array}\right) \frac{(-2 \log |z|)^{a+b-1-k}}{(a+b-1-k) !} \operatorname{Li}_{k}(z) \\
& +(-1)^{b-1} \sum_{k=b}^{a+b-1}\left(\begin{array}{c}
k-1 \\
b-1
\end{array}\right) \frac{(-2 \log |z|)^{a+b-1-k}}{(a+b-1-k) !}\left(\operatorname{Li}_{k}(z)\right)^{*} .
\end{aligned}
$$

These functions satisfy the complex conjugation relation $D_{a, b}(z)^{*}=D_{b, a}(z)$, and we define their weight to be $a+b-1$. In particular, the function $D_{a, a}(z)$ with $a \in \mathbb{N}$ has weight $2 a-1$ and is real and single-valued on $\mathbb{C} \backslash\{0,1\}$. It is given by a finite linear combination of the $\operatorname{Li}_{k}(z)$-functions

$$
D_{a, a}(z)=2 \Re \mathrm{e}\left((-1)^{a-1} \sum_{k=0}^{a-1}\left(\begin{array}{c}
k+a-1 \\
a-1
\end{array}\right) \frac{(-2 \log |z|)^{a-1-k}}{(a-1-k) !} \operatorname{Li}_{a+k}(z)\right),
$$

and will play an important role in the sequel of this paper. The following are examples at low weights. At weight one, we have,

$$
D_{1,1}(z)=-2 \log |1-z|^{2}
$$


which is manifestly single-valued. At weight 2 we have

$$
\begin{aligned}
& D_{1,2}(z)=2 i D(z)+2 \log |z| \log |1-z|, \\
& D_{2,1}(z)=-2 i D(z)+2 \log |z| \log |1-z|,
\end{aligned}
$$

where $D(z)$ is the Bloch-Wigner dilogarithm of (2.8) which is single-valued on $\mathbb{C} \backslash\{0,1\}$. At weight 3 we have

$$
\begin{aligned}
& D_{1,3}(z)=2(\log |z|)^{2} \operatorname{Li}_{1}(z)-2 \log |z| \operatorname{Li}_{2}(z)+2 \Re \mathrm{e}\left(\operatorname{Li}_{3}(z)\right), \\
& D_{2,2}(z)=4 \log |z| \Re \mathrm{e}\left(\operatorname{Li}_{2}(z)\right)-4 \Re \mathrm{e}\left(\operatorname{Li}_{3}(z)\right) .
\end{aligned}
$$

It is not difficult to check that these functions are algebraically independent.

2.3. Multiple polylogarithms. The multi-variable polylogarithm $\operatorname{Li}_{a_{1}, \ldots, a_{r}}\left(z_{1}, \ldots, z_{r}\right)$ is referred to as a multiple polylogarithm and was defined by [21,22]

$$
\operatorname{Li}_{a_{1}, \ldots, a_{r}}\left(z_{1}, \ldots, z_{r}\right)=\sum_{0<m_{1}<\cdots<m_{r}} \prod_{i=1}^{r} \frac{z_{i}^{m_{i}}}{m_{i}^{a_{i}}},
$$

with $a_{i} \in \mathbb{N}$ for $1 \leq i \leq r$, and with $\left|z_{i}\right| \leq 1(1 \leq i \leq r-1)$ and $\left|z_{r}\right|<1$. When $a_{i} \in \mathbb{N} \geq 2$ for all $1 \leq i \leq r$ this function is defined for $\left|z_{i}\right| \leq 1$ for $1 \leq i \leq r$.

The weight of a multiple polylogarithm is the sum of its indices $\sum_{i=1}^{r} a_{i}$ and its depth is the number of indices, $r$. The classical polylogarithm functions $\operatorname{Li}_{a}(z)$ are special cases with depth $r=1$.

The multiple polylogarithms in (2.14) satisfy two kinds of differential relations. One of these reduces the weight by one but leaves the depth unchanged

$$
z_{i} \frac{\partial}{\partial z_{i}} \operatorname{Li}_{a_{1}, \ldots, a_{r}}\left(z_{1}, \ldots, z_{r}\right)=\operatorname{Li}_{a_{1}, \ldots, a_{i}-1, \ldots, a_{r}}\left(z_{1}, \ldots, z_{r}\right) \quad 1 \leq i \leq r,
$$

while the other reduces both the weight and the depth by one

$$
\left(1-z_{r}\right) \frac{\partial}{\partial z_{r}} \operatorname{Li}_{a_{1}, \ldots, a_{r-1}, 1}\left(z_{1}, \ldots, z_{r}\right)=\operatorname{Li}_{a_{1}, \ldots, a_{r-1}}\left(z_{1}, \ldots, z_{r-1} z_{r}\right) .
$$

Together with the initial conditions $\operatorname{Li}_{a_{1}, \ldots, a_{r}}(0, \ldots, 0)=0$ these differential equations determine the multiple polylogarithms by multiple integration. This leads to Chen iterated integrals [23] which endow the space of multiple polylogarithms with a shuffle algebra. Since we will not make use of this construction we refer to [19] for details and references. For additional reference, we note that these properties have been implemented in the algebraic program Hyperint by Erik Panzer [24] and in MPL by Christian Bogner in [25].

From the series representation one derives the stuffle relations as described in [26], and implemented in $[27,28]$. The stuffle relation of two multiple polylogarithms is given 
by

$$
\operatorname{Li}_{a_{1}, \ldots, a_{r}}\left(x_{1}, \ldots, x_{r}\right) \operatorname{Li}_{b_{1}, \ldots, b_{s}}\left(y_{1}, \ldots, y_{s}\right)=\sum_{k=\max (r, s)}^{r+s} \operatorname{Li}_{c_{1}, \ldots, c_{k}}\left(z_{1}, \ldots, z_{k}\right),
$$

where the sum is over all the sequences $c(k):=\left(c_{1}, \ldots, c_{k}\right)$ that arise in the stuffle product of the sequences $a(r):=\left(a_{1}, \ldots, a_{r}\right)$ and $b(s):=\left(b_{1}, \ldots, b_{s}\right)$. The stuffle product, denoted $*$, is a commutative product defined recursively by

- $\left(a_{1}, \ldots, a_{r}\right) *\{\}=\left(a_{1}, \ldots, a_{r}\right)$ where \{\} is the empty sequence

- $\left(a_{1}, \ldots, a_{r}\right) *\left(b_{1}, \ldots, b_{s}\right)=a_{1} \cdot\left(\left(a_{2}, \ldots, a_{r}\right) *\left(b_{1}, \ldots, b_{s}\right)\right)+b_{1} \cdot\left(\left(a_{1}, \ldots, a_{r}\right) *\right.$ $\left.\left(b_{2}, \ldots, b_{s}\right)\right)+\left(a_{1}+b_{1}\right) \cdot\left(\left(a_{2}, \ldots, a_{r}\right) *\left(b_{2}, \ldots, b_{s}\right)\right)$

- $x \cdot\left(a_{1}, \ldots, a_{r}\right)=\left(x, a_{1}, \ldots, a_{r}\right)$ is the concatenation operation

The argument $z_{i}$ associated with the index $c(k)_{i}=a(k)_{i}+b(k)_{i}$ is obtained as follows:

- if $a(k)_{i} \neq 0$ and $b(k)_{i}=0$ then $z_{i}=x_{i}$;

- if $a(k)_{i}=0$ and $b(k)_{i} \neq 0$ then $z_{i}=y_{i}$;

- if $a(k)_{i} \neq 0$ and $b(k)_{i} \neq 0$ then $z_{i}=x_{i} y_{i}$.

Note that since we have assumed that $a_{i} \geq 1$ for all $i=1, \ldots, r$ and $b_{j} \geq 1$ for all $j=1, \ldots, s$, the case where both $a(k)_{i}=0$ and $b(k)_{i}=0$ cannot arise.

We illustrate this with two examples that will be useful later. The first example is the product of two depth-1 polylogarithms

$$
\operatorname{Li}_{a}(x) \operatorname{Li}_{b}(y)=\operatorname{Li}_{a+b}(x y)+\operatorname{Li}_{a, b}(x, y)+\operatorname{Li}_{b, a}(y, x) .
$$

The second example is the product of a depth- 2 multiple polylogarithm by a depth-1 polylogarithm

$$
\begin{aligned}
\operatorname{Li}_{a, b}(x, y) \operatorname{Li}_{c}(z)=\operatorname{Li}_{a+c, b} & (x z, y)+\operatorname{Li}_{a, b+c}(x, y z) \\
& +\operatorname{Li}_{a, b, c}(x, y, z)+\operatorname{Li}_{a, c, b}(x, z, y)+\operatorname{Li}_{c, a, b}(z, x, y) .
\end{aligned}
$$

2.4. Single-valued multiple polylogarithms. The special case of single-variable multiple polylogarithms, which have the form

$$
\operatorname{Li}_{a_{1}, \ldots, a_{r}}(z)=\operatorname{Li}_{a_{1}, \ldots, a_{r}}(1, \ldots, 1, z),
$$

have monodromies around $z=0$ and $z=1$. However single valued versions on $\mathbb{C} \backslash\{0,1\}$ of such functions were constructed by Francis Brown in [6,8]. They are obtained by appropriate linear combinations of product single-variable multiple polylogarithms and their complex conjugate to define functions on $\mathbb{C} \backslash\{0,1\}$ without monodromies. These combinations are examples of single-valued multiple polylogarithms. A construction of single-valued multiple polylogarithms with more than one variable has been given in [29], which appeared some months after the first version of this paper appeared on the arXiv. 
2.5. Multiple zeta values and single-valued multiple zeta values. Multiple zeta values [30] provide a natural generalisation of Riemann zeta values. The multiple zeta function may be defined by the following multiple sums for $\Re \mathrm{e}\left(a_{i}\right)>1$,

$$
\zeta\left(a_{1}, \ldots, a_{r}\right)=\sum_{0<n_{1}<\cdots<n_{r}} \prod_{i=1}^{r} \frac{1}{n_{i}^{a_{i}}},
$$

and analytically continued to $a_{i} \in \mathbb{C}$. For $a_{i} \in \mathbb{N}$, and $a_{r}>1$, the quantities $\zeta\left(a_{1}, \ldots, a_{r}\right)$ give multiple zeta values. The multiple polylogarithm evaluated with all its arguments equal to 1 is a multiple zeta value given by

$$
\operatorname{Li}_{a_{1}, \ldots, a_{r}}(1, \ldots, 1)=\zeta\left(a_{1}, \ldots, a_{r}\right)
$$

when $a_{i} \in \mathbb{N}$ with $a_{r}>1 .^{2}$

The basis of multiple zeta values has been understood in terms of periods on the Riemann sphere with marked points [31]. This interpretation also arises naturally in the analysis of the low energy expansion of string theory tree amplitudes [32-34]. The stuffle and shuffle relations for multiple polylogarithms lead to a wealth of algebraic relations between multiple zeta values.

The single-valued multiple zetas are obtained by setting the arguments of singlevalued multiple polylogarithms to 1 . For example,

$$
\begin{aligned}
& D_{1,2}(1)=D_{2,1}(1)=0 \\
& D_{1,3}(1)=2 \zeta(3) \\
& D_{2,2}(1)=-4 \zeta(3)
\end{aligned}
$$

It is not difficult to check that these functions have the properties

$$
\begin{array}{lll}
D_{a, b}(1) & =0 & \\
D_{a, b}(1) \in \zeta(a+b-1) \times \mathbb{Z} & & a+b \in 2 \mathbb{N} .1
\end{array}
$$

This has led Brown to define single-valued zeta values $\zeta_{s v}$ that are given by [8]

$$
\begin{array}{rlr}
\zeta_{s v}(2 n) & =0 \\
\zeta_{s v}(2 n+1) & =2 \zeta(2 n+1) . &
\end{array} \quad n \in \mathbb{N}
$$

It is important to appreciate that the definition of a single-valued polylogarithm on $\mathbb{C} \backslash\{0,1\}$ is not unique for weight greater than two. ${ }^{3}$ Different definitions, when evaluated at 1 , gives rise to a different rational linear combination of single-valued multiple zeta. The functions $D_{a, b}(z)$ in (2.9) give particular examples of single-valued multiple polylogarithms.

\footnotetext{
${ }^{2}$ When $a_{r}=1$ the expression diverges and has to be regularised, but this case will not concern us in the sequel.

${ }^{3} \mathrm{~A}$ discussion of the general properties of such functions and the relations between various equivalent definitions may be found, for example, in [6]. This paper provides a canonical choice of single-valued multiple polylogarithms.
} 
2.6. The basis of single-valued multiple zetas. The dimension $d_{w}$ of the subspace of multiple zeta values of weight $w$ in the ring over $\mathbb{Q}$ of multiple zeta values is conjecturally given by the expansion of a rational function $[30,35,36]$

$$
\sum_{w=0}^{\infty} d_{w} x^{w}=\frac{1}{1-x^{2}-x^{3}} .
$$

The dimension $d_{w}^{s v}$ of the subspace of weight $w$ in the ring over $\mathbb{Q}$ of single-valued multiple-zeta values is smaller than $d_{w}$ [8]. For example, at weight 11 the basis of ordinary multiple zeta values has dimension 9 and is composed of

$$
\begin{aligned}
& \zeta(3,5,3), \zeta(3,5) \zeta(3), \zeta(3)^{2} \zeta(5), \zeta(11), \\
& \zeta(2) \zeta(3)^{3}, \zeta(2)^{4} \zeta(3), \zeta(2)^{3} \zeta(5), \zeta(2)^{2} \zeta(7), \zeta(2) \zeta(9) .
\end{aligned}
$$

Since by definition we have $\zeta_{s v}(2)=0$ all the basis elements for multiple zeta values on the second line are (conjecturally) mapped to zero as elements of single-valued multiple zetas. At weight 11 a further reduction happens since according to [8] we have the relation

$$
\zeta_{s v}(3,5,3)=2 \zeta(3,5,3)-2 \zeta(3) \zeta(3,5)-10 \zeta(3)^{2} \zeta(5)
$$

and since $\zeta_{s v}(3,5)=-10 \zeta(3) \zeta(5)$, the basis of single-valued multiple zetas at weight 11 has dimension 3 and is composed of

$$
\zeta_{s v}(3,5,3), \zeta_{s v}(3)^{2} \zeta_{s v}(5), \zeta_{s v}(11) \text {. }
$$

Corrollary 7.4 of [8] gives the dimensions $d_{w}^{s v}$ of the subspace of single-valued multiple zetas of weight $w$

$$
\sum_{w=0}^{\infty} d_{w}^{s v} x^{w}=\prod_{n=0}^{\infty}\left(1-x^{2 n+1}\right)^{-\ell_{2 n+1}}
$$

where the positive integers $\ell_{n}$ are defined by the following product relation,

$$
\prod_{n=1}^{\infty}\left(1-x^{n}\right)^{\ell_{n}}=1-x^{2}-x^{3} .
$$

Note that, up to weight 12 , the non-zero values for $\ell$ are $\ell_{2}=\ell_{3}=\ell_{5}=\ell_{7}=\ell_{8}=\ell_{9}=$ $\ell_{10}=1$ and $\ell_{11}=\ell_{12}=2$, so that the non-zero values for $d_{w}^{s v}$ are $d_{3}^{s v}=d_{5}^{s v}=d_{6}^{s v}=d_{7}^{s v}=$ $d_{8}^{s v}=1$, while $d_{9}^{s v}=d_{10}^{s v}=2$ and $d_{11}^{s v}=3$ in agreement with our earlier calculation. Single-valued multiple zeta values are very efficiently obtained using Schnetz' Maple routines [37]. ${ }^{4}$

\footnotetext{
${ }^{4}$ The procedure in [37] presents a Feynman diagram description of single-valued multiple polylogarithms reminiscent of the description to be presented in this paper. However, the discussion there is in the context of quantum field theories that are quite different from the two-dimensional conformal field theory that we are considering, and refers to genus-zero functions, which need special treatment.
} 
We will give an example in section 4.3 of how such single-valued multiple zetas enter into the Laurent expansions of the modular graph functions. They have already appeared in Feynman diagrams in quantum field theory [7] and in the low energy expansion of closed string tree level amplitudes $[4,5]$.

2.7. Single-valued elliptic polylogarithms. In the main part of this paper we will consider generalisations of polylogarithms and multiple polylogarithms that are defined on a general elliptic curve, that enters, for example, into the integrand of the genus one closed string amplitude. An elliptic curve $\mathcal{E}$ can be described either as $\mathcal{E} \simeq \mathbb{C} /(\mathbb{Z}+\tau \mathbb{Z})$ where $\tau$ is the period ratio or as $\mathcal{E} \simeq \mathbb{C}^{\times} / q^{\mathbb{Z}}$ where $q=\exp (2 i \pi \tau)$, and $\mathbb{C}^{\times}$is the multiplicative group of non-zero complex numbers. A point $P$ on the elliptic curve is represented as $\zeta(P)=\exp (2 i \pi(u \tau+v)) \in \mathbb{C}^{\times} / q^{\mathbb{Z}}$ where $u$ and $v$ are cartesian coordinates in $[0,1]$. The real part of the period ratio is denoted by $\tau_{1}=\Re \mathrm{e}(\tau)$ and its imaginary part by $\tau_{2}=\Im \mathrm{m}(\tau)$.

We will make extensive use of the elliptic polylogarithms that were constructed by Zagier [20]. These can be expressed as infinite sums over positive integers $n$ of images of the polylogarithms $\operatorname{Li}_{k}(z)$ under the translations $z \rightarrow z+n \tau$. To be explicit, these funtions are defined by

$$
D_{a, b}(q ; \zeta)=\sum_{n \geq 0} D_{a, b}\left(q^{n} \zeta\right)+(-1)^{a+b} \sum_{n \geq 1} D_{a, b}\left(q^{n} / \zeta\right)+\frac{\left(4 \pi \tau_{2}\right)^{a+b-1}}{(a+b) !} B_{a+b}(u)
$$

where the function $D_{a, b}(x)$ was defined in (2.9), $B_{n}$ is the $n$-th Bernoulli polynomial, and $\zeta=e^{2 \pi i(u \tau+v)}$ corresponds to a point on the torus with modulus $q=e^{2 \pi i \tau}$ for $u, v \in[0,1]$. Zagier showed that if $a$ and $b$ are two positive integers, this function is equal to the double sum

$$
D_{a, b}(q ; \zeta)=\frac{\left(2 i \tau_{2}\right)^{a+b-1}}{2 i \pi} \sum_{(m, n) \neq(0,0)} \frac{e^{2 i \pi(n u-m v)}}{(m \tau+n)^{a}(m \bar{\tau}+n)^{b}} .
$$

The function $D_{a, b}(q ; \zeta)$ is invariant under $\zeta \rightarrow q \zeta$ since the expression in (2.33) is invariant under the shifts $u \rightarrow u+1$ and $v \rightarrow v+1$, therefore this is a function on the elliptic curve. Furthermore, under the action of $S L(2, \mathbb{Z})$

$$
\begin{aligned}
\tau & \rightarrow \frac{\alpha \tau+\beta}{\gamma \tau+\delta} \quad \alpha \delta-\beta \gamma=1, \quad \alpha, \beta, \gamma, \delta \in \mathbb{Z} \\
(u, v) & \rightarrow(\delta u-\gamma v,-\beta u+\alpha v)
\end{aligned}
$$

$D_{a, b}(q ; \zeta)$ transforms as a modular form of weight $(1-b, 1-a)$, namely,

$$
D_{a, b}(q ; \zeta) \rightarrow(\gamma \tau+\delta)^{1-b}(\gamma \bar{\tau}+\delta)^{1-a} D_{a, b}(q ; \zeta)
$$


The special case $a=b$ will be of particular importance in this paper and is given by

$$
D_{a, a}(q ; \zeta)=\sum_{n \geq 0} D_{a, a}\left(q^{n} \zeta\right)+\sum_{n \geq 1} D_{a, a}\left(q^{n} / \zeta\right)+\frac{\left(4 \pi \tau_{2}\right)^{2 a-1}}{(2 a) !} B_{2 a}(u),
$$

where $D_{a, a}(x)$ is given by (2.10). The elliptic polylogarithms defined in this manner are manifestly single-valued, as follows from their form in (2.33).

2.8. Elliptic multiple polylogarithms. Elliptical generalisations of multiple polylogarithms have been defined in [14]. These are holomorphic functions of $q$. These are of relevance to loop amplitudes in open string theory [15] as well as arising in dimensionally regularised Feynman graphs in quantum field theory at two loops [38-40] and three loops [41]. References [38,41] give a motivic approach to these objects. We will not review the detailed form of these functions since they do not arise in the rest of this paper.

It will be established later in this paper that we may define single-valued elliptic multiple polylogarithms as integrals of products of $D_{1,1}(q ; \zeta)$ factors over the torus, with one argument left unintegrated. Modular graph functions may then be described as the values of single-valued elliptic multiple polylogarithms with the unintegrated argument set equal to 1 . The terminology single-valued arises because single-valued elliptic multiple polylogarithms have no monodromy as functions of their unintegrated arguments. This mimics the terminology used for single-valued multiple zeta values, which are the values of single-valued multiple polylogarithms with arguments set equal to 1 .

The single-valued functions that we will describe should be distinguished from the elliptic multiple polylogarithms that arise in [14] (mentioned above), which are holomorphic functions of $q$. It would be very interesting to discover a precise relationship between the single-valued and non-single-valued elliptical multiple polylogarithms.

\section{Feynman graphs associated With a torus}

Our motivation for considering modular graph functions originated from the study of the low energy expansion of the genus-one four-graviton amplitude in Type II superstring theory. The graphs generated in this expansion have up to four vertices joined by the scalar Green function on the torus, as will be reviewed below. However, the structure of such graphs generalises in an obvious fashion to graphs with an arbitrary number of vertices, and it is these general graphs that will be considered in this section and sections 4, 5, and 6 below. Actually, this obvious generalisation will not quite encompass all the contributions that can arise in the $N$-string amplitudes at genus-one for $N>4$, as was discussed to a limited extent in [42]. We will comment on the structure of such graphs in section 7 . 
The four-graviton amplitude at genus one is given by an integral over the moduli space $\mathcal{M}_{1}$ of a torus $\Sigma$ of a partial amplitude $\mathcal{B}_{4}$ evaluated at fixed modulus. A torus $\Sigma$ with modulus $\tau$ may be represented in the complex plane by $\mathbb{C} /(\mathbb{Z} \tau+\mathbb{Z})$ and parametrised by a complex coordinate $z=u \tau+v$ or equivalently by two real coordinates $u, v \in \mathbb{R} / \mathbb{Z}$. The volume form on $\Sigma$ is normalised to $d^{2} z=i d z \wedge d \bar{z} / 2=$ $-\tau_{2} d u \wedge d v$. The moduli space $\mathcal{M}_{1}$ of orientable genus-one Riemann surfaces (or tori) may be represented by a fundamental domain for the action of $P S L(2, \mathbb{Z})$ on the Poincaré upper half plane, which may be parametrised by $\tau=\tau_{1}+i \tau_{2}$ with $\tau_{1}, \tau_{2} \in \mathbb{R}$ and $\mathcal{M}_{1}=\left\{\tau, 0<\tau_{2},|\tau|>1,\left|\tau_{1}\right|<\frac{1}{2}\right\}$ and the contribution from the boundaries $\left\{\tau, 0<\tau_{2},|\tau| \geq 1, \tau_{1}=-\frac{1}{2}\right\}$ and $\left\{\tau, 0<\tau_{2},|\tau|=1,-\frac{1}{2}<\tau_{1} \leq 0\right\}$. We define the following partial amplitude $\mathcal{B}_{N}\left(s_{i j} \mid \tau\right)$ for arbitrary $N$

$$
\mathcal{B}_{N}\left(s_{i j} \mid \tau\right)=\prod_{n=1}^{N} \int_{\Sigma} \frac{d^{2} z_{n}}{\tau_{2}} \exp \left(\sum_{1 \leq i<j \leq N} s_{i j} G\left(z_{i}-z_{j} \mid \tau\right)\right)
$$

The scalar Green function $G\left(z_{i}-z_{j} \mid \tau\right)$ on the torus will be discussed in the subsection below. The parameters $s_{i j}$ are related to the momenta $k_{i}$ of $N$ gravitons, for $i=$ $1, \ldots, N$. Each momentum $k_{i}$ is a null-vector in 10-dimensional Minkowski space-time $\mathbb{R}^{10}$ so that $k_{i} \cdot k_{i}=0$. The relation between the $s_{i j}$ and $k_{i}$ is given by $s_{i j}=-\ell_{s}^{2} k_{i} \cdot k_{j} / 2$, where $\ell_{s}$ is the string length. The null-vector condition on $k_{i}$ and overall momentum conservation $\sum_{i=1}^{N} k_{i}=0$ impose the following relations between the parameters $s_{i j}$

$$
s_{i i}=0, \quad \quad \sum_{j=1}^{N} s_{i j}=0, \quad i=1, \ldots, N
$$

Actually, any partition of the sum over momenta into two disjoint sets will give rise to extra linear partition relations between the variables $s_{i j}$. As a result, for $N \leq 3$, we have $s_{i j}=0$ for all $1 \leq i, j \leq 3$ and thus $\mathcal{B}_{1}=\mathcal{B}_{2}=\mathcal{B}_{3}=1$. The first nontrivial case is the four-graviton amplitude with $N=4$, and extra partition relations $s_{12}=s_{34}, s_{23}=s_{14}, s_{13}=s_{24}=-s_{12}-s_{14}$ resulting from partitioning the momenta into pairs.

The partial amplitude $\mathcal{B}_{N}\left(s_{i j} \mid \tau\right)$, with the parameters $s_{i j}$ subject to the relations (3.2), is well-defined for any value of $N$, and will be taken here as the generating function for modular graph functions, although it is only when $N=4$ that $\mathcal{B}_{N}\left(s_{i j} \mid \tau\right)$ 
corresponds precisely to the partial closed superstring amplitude. ${ }^{5}$ The restrictions imposed by the extra partition relations between the parameters $s_{i j}$ will be left unenforced, as they may be imposed easily, if needed, when constructing the partial amplitudes for actual string processes.

3.1. The scalar Green function. A scalar Green function $G(z \mid \tau)$ is defined to be the inverse of the Laplace operator $\Delta=4 \partial_{\bar{z}} \partial_{z}$ on the torus, $\Sigma$. Since $\mathcal{B}_{N}$ is invariant under a $z$-independent shift of the Green function $G(z \mid \tau)$ because of momentum conservation, we may restrict the Green function to have vanishing integral on $\Sigma$. Therefore, $G(z \mid \tau)$ obeys the following equations

$$
\Delta G(z \mid \tau)=-4 \pi \delta^{(2)}(z)+\frac{4 \pi}{\tau_{2}} \quad \int_{\Sigma} d^{2} z G(z \mid \tau)=0 .
$$

This determines the modular invariant formula for $G$ in terms of the Jacobi $\vartheta_{1}$-function and the Dedekind $\eta$-function

$$
G(z \mid \tau)=-\ln \left|\frac{\vartheta_{1}(z \mid \tau)}{\eta(\tau)}\right|^{2}-\frac{\pi}{2 \tau_{2}}(z-\bar{z})^{2} .
$$

Equivalently, the Green function may be expressed as a Fourier sum in terms of the real variables $u, v \in \mathbb{R} / \mathbb{Z}$ defined by $z=u \tau+v$

$$
G(z \mid \tau)=\sum_{(m, n) \neq(0,0)} \frac{\tau_{2}}{\pi|m \tau+n|^{2}} e^{2 i \pi(m v-n u)} .
$$

The integers $m, n$ parametrise the discrete momenta $p=m \tau+n \in \mathbb{Z} \tau+\mathbb{Z}$ of the Fourier modes on the torus.

To make contact with polylogarithms, we change variables for both the modulus $\tau$ and the coordinate $z$ on the torus, to new variables defined in terms of $\tau$ and $z$ by

$$
q=e^{2 \pi i \tau} \quad \zeta=e^{2 \pi i z}=e^{2 \pi i(u \tau+v)}
$$

The condition $0<\tau_{2}$ guarantees that $|q|<1$, while periodicity of $\zeta$ in $v$ shows that $\Sigma$ is parametrised by $\zeta \in \mathbb{C}^{\times} / q^{\mathbb{Z}}$. Comparing the expression for $D_{a, b}(q ; \zeta)$ of $(2.33)$ for $a=b=1$ with the Fourier sum in (3.6), we are led to the following identification $[17,20]$

$$
D_{1,1}(q ; \zeta)=G(z \mid \tau) \text {. }
$$

\footnotetext{
${ }^{5}$ See the discussion in section 7 for some comments concerning the $N>4$ amplitude. The genus-one four-graviton amplitude is given by

$$
\mathcal{A}\left(\epsilon_{i}, k_{i}\right)=2 \pi \kappa_{10}^{2} \mathcal{R}^{4} \mathcal{B}_{4}\left(s_{12}, s_{13}\right)
$$

where $\kappa_{10}^{2}$ is the ten-dimensional Newton constant and the graviton polarisations, which appear in the linearised curvature tensor $\mathcal{R}$, are denoted by $\epsilon_{i}$. The $\alpha^{\prime}$ expansion of $\mathcal{B}_{4}\left(s_{12}, s_{13}\right)$ only involves contributions from products of the Green functions connecting at most $N=4$ vertices on the worldsheet.
} 
Similarly, the expression $D_{1,1}\left(q ; \zeta_{i} / \zeta_{j}\right)$ will equal the Green function $G\left(z_{i}-z_{j} \mid \tau\right)$ on the torus between the points $i$ and $j$, with $\zeta_{i} / \zeta_{j}=e^{2 i \pi\left(z_{i}-z_{j}\right)}$. In view of this identity, we shall often refer to $D_{1,1}(q ; \zeta)$ as the Green function as well.

Importantly for the subsequent discussions, the Green function $D_{1,1}(q ; \zeta)$ admits a simple expression in terms of polylogarithms $2 \Re \mathrm{eLi}_{1}(\cdot)$, which are single-valued on $\mathbb{C} \backslash\{0,1\}$, evaluated on an elliptic curve

$$
D_{1,1}(q ; \zeta)=2 \Re \mathrm{e}\left(\sum_{n \geq 0} \operatorname{Li}_{1}\left(q^{n} \zeta\right)+\sum_{n \geq 1} \operatorname{Li}_{1}\left(q^{n} / \zeta\right)+\pi \tau_{2} B_{2}(u)\right),
$$

where $\zeta=q^{u} e^{2 i \pi v}$ with $u, v \in[0,1]$, and $B_{2}(u)$ is the second Bernoulli polynomial.

3.2. Modular graph functions. The Fourier representation of $G(z \mid \tau)$ makes it transparent that the Green function $G(z \mid \tau)$ is invariant under the modular transformations of (2.34). As a result, the function $\mathcal{B}_{N}\left(s_{i j} \mid \tau\right)$ is also modular invariant when the variables $s_{i j}$ are left unchanged under the modular transformation. The series expansion of $\mathcal{B}_{N}\left(s_{i j} \mid \tau\right)$ in powers of $s_{i j}$ has a finite radius of convergence, and may be organised graphically in what physicists refer to as Feynman graphs, in this case for a conformal scalar field on the torus. Each term in this expansion corresponds to a specific graph, and will evaluate to a specific non-holomorphic modular function, whence the terminology modular graph function.

The building block of the modular graphs is the scalar Green function $G\left(z_{i}-z_{j} \mid \tau\right)$, which we associate with an edge in the graph between vertices $z_{i}$ and $z_{j}$. Alternatively, and more conveniently for application to polylogarithms, the scalar Green function may be set to the function $D_{1,1}\left(q ; \zeta_{i} / \zeta_{j}\right)$. We have the following graphical representation

$$
\zeta_{i} \quad \zeta_{j}=D_{1,1}\left(q ; \zeta_{i} / \zeta_{j}\right)=G\left(z_{i}-z_{j} \mid \tau\right) .
$$

The Green function between two vertices $\zeta_{i}$ and $\zeta_{j}$ may enter into a given graph raised to an integer power $n$, for which we shall use the following graphical representation,

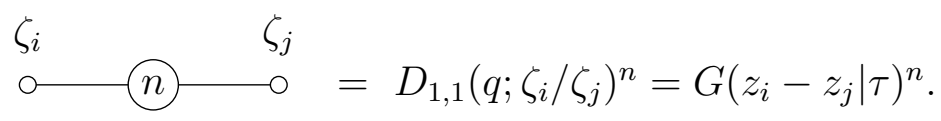

Finally, the integration at one vertex over a product of Green functions ending at that vertex will be denoted by a filled black dot, in contrast with an unintegrated vertex which will be represented by an unfilled dot. The basic ingredient in the graphical notation is depicted in the graph below

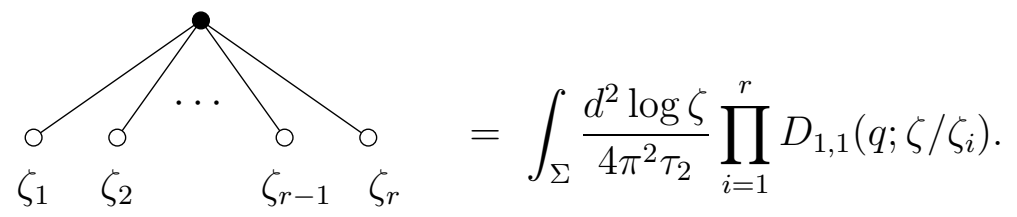


The low energy expansion of $\mathcal{B}_{N}$ is obtained simply by expanding the exponential in (3.1) in powers of $s_{i j}$, or equivalently by expanding in powers of $\ell_{s}^{2}$, while keeping the momenta $k_{i}$ fixed. The coefficient of the term which is homogeneous of degree $w$ in the $s_{i j}$ variables is given by a sum over all possible graphs with $w$ Green functions joining at most $N$ vertices, as studied for the special case of $N=4$ in $[9,11,17,18]$. Each weight $w$ graph $\Gamma_{w, N}$ is evaluated by the integration of the product of Green functions over the position of the vertices $\zeta_{i}$ on the torus $\Sigma$. When no confusion is expected to arise, we shall abbreviate $\Gamma_{w, N}$ by simply $\Gamma$. The resulting function will be denoted $I_{\Gamma}(q)$ and is given by

$$
I_{\Gamma}(q)=\prod_{k=1}^{N} \int_{\Sigma} \frac{d^{2} \log \zeta_{k}}{4 \pi^{2} \tau_{2}} \prod_{1 \leq i<j \leq N} D_{1,1}\left(q ; \zeta_{i} / \zeta_{j}\right)^{n_{i j}},
$$

where $N$ is the number of vertices and $n_{i j}$ is the number of Green functions joining the vertices $i$ and $j$. The numbers $n_{i j}$ are the entries of the adjacency matrix of the graph $\Gamma=\Gamma_{w, N}$. By construction, the integral $I_{\Gamma}(q)$ is modular invariant, as it arises from the expansion in powers of $s_{i j}$ of the modular invariant generating function $\mathcal{B}_{N}\left(s_{i j} \mid \tau\right)$, and therefore associates with a graph $\Gamma$ a non-holomorphic modular function $I_{\Gamma}(q)$ of $q$ and $\bar{q}$.

An alternative evaluation of the modular graph function $I_{\Gamma}(q)$ is obtained by using the Fourier representation

$$
D_{1,1}(q ; \zeta)=\sum_{(m, n) \neq(0,0)} \frac{\tau_{2}}{\pi|m \tau+n|^{2}} e^{2 i \pi(m v-n u)},
$$

where $\zeta=e^{2 \pi i(u \tau+v)}$. The integration over $\Sigma$ of the position $\zeta_{i}$ of the vertex $i$ enforces momentum conservation on all the Green functions that enter the vertex $i$. Carrying out the integrations over $\Sigma$ for all the vertex positions $\zeta_{i}$ for $i=1, \ldots, N$ gives the constrained multiple sum representation for the graphs, as was studied already earlier for the case $N=4$ in $[9-11,17,18]$. The general form of the sum may be schematically represented as follows

$$
I_{\Gamma}(q)=\sum_{p_{1}, \ldots, p_{w} \in \mathbb{Z} \tau+\mathbb{Z}}^{\prime} \prod_{\alpha=1}^{w} \frac{\tau_{2}}{\pi\left|p_{\alpha}\right|^{2}} \prod_{i=1}^{N} \delta\left(\sum_{\alpha=1}^{w} C_{i \alpha} p_{\alpha}\right) .
$$

Here, the prime above the summation symbol indicates that the sums over $p$ exclude the value 0 ; the Kronecker $\delta$ symbol takes the value 1 when its argument vanishes and zero otherwise; the coefficients $C_{i \alpha}$ are given as follows

$$
C_{i \alpha}=\left\{\begin{array}{cc} 
\pm 1 & \text { if edge } \alpha \text { ends on vertex } i \\
0 & \text { otherwise }
\end{array}\right.
$$


the sign being determined by the orientation of the momenta. Note that, given the weight $w$, all the information on the graph $\Gamma$ is encoded in the coefficients $C_{i \alpha}$, the other parts of the sum in (3.12) being the same for all $w$. This momentum representation of the modular graph function clearly confirms its modular invariance.

3.3. Single-valued nature of the modular graph functions. In order to understand the statement that the modular graph functions are single-valued, we now show that they are naturally expressed in terms of elliptic analogues of single-valued multiple polylogarithms. We will call these functions single-valued elliptic multiple polylogarithms. We will demonstrate that the function $I_{\Gamma}(q)$ is a special value of a more general function, $I_{\Gamma}(q ; \zeta)$, associated with the graph $\Gamma=\Gamma_{w, N}$ when all but one of the vertices, say vertex $j$, are integrated and the lines meeting the unintegrated vertex $j$ are made to terminate at two separate points, $\zeta_{j}$ and $\zeta_{j}^{\prime}$. By translation invariance on the torus, we may set $\zeta_{j}^{\prime}=1$ without loss of generality. The function $I_{\Gamma}(q, \zeta)$ has no monodromies in $\zeta$ and is therefore single-valued on the torus.

To be as concrete as possible, we shall concentrate here on the case of $N=4$, but our considerations may be easily generalised to other values of $N$. An arbitrary graph $\Gamma=\Gamma_{w, 4}$ of weight $w$ and with four vertices may be labeled as follows

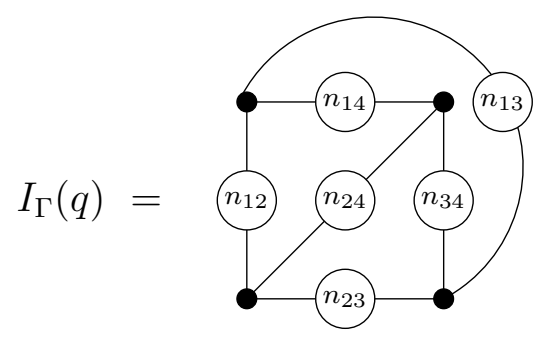

The resulting elliptic function $I_{\Gamma}\left(q ; \zeta_{1}\right)$ is illustrated by the graph below with five vertices, of which two are unintegrated. The unintegrated vertex corresponds here to the vertex $j=1$ in the figure

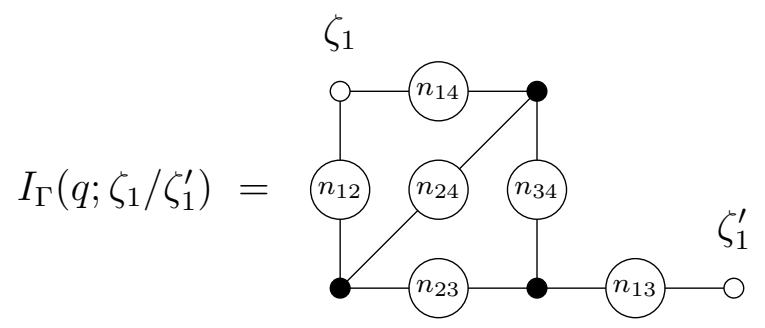

The black dots represent integrated vertices, while the white dots continue to represent unintegrated vertices. To such a graph we associate an elliptic function that results from the integration over the remaining three vertices, which here are $k=2,3,4$

$$
I_{\Gamma}(q ; \zeta)=\prod_{k=2}^{4} \int_{\Sigma} \frac{d^{2} \log \zeta_{k}}{4 \pi^{2} \tau_{2}} \prod_{1 \leq i<j \leq 4} D_{1,1}\left(q ; \zeta_{j} / \zeta_{i}\right)^{n_{i j}}\left(\frac{D_{1,1}\left(q ; \zeta_{1}^{\prime} / \zeta_{3}\right)}{D_{1,1}\left(q ; \zeta_{1} / \zeta_{3}\right)}\right)^{n_{13}}
$$


The function $I_{\Gamma}(q ; \zeta)$ depends only on the ratio $\zeta=\zeta_{1} / \zeta_{1}^{\prime}$ due to translation invariance on the torus. Since the Green function $D_{1,1}(q ; \zeta)$ is a single-valued function of $\zeta$ the function $I_{\Gamma}(q ; \zeta)$ is a single-valued function of $\zeta$. Translation invariance implies that the full integral (3.10) is recovered by identifying the points $\zeta_{1}$ and $\zeta_{1}^{\prime}$ in the above graph, in other words, by setting $\zeta=1$. This is easily shown using the representation of (3.12) in which the discrete momentum is preserved at each vertex.

3.4. Proposition. Any modular graph function $I_{\Gamma}(q)$ may be obtained as the value of a single-valued elliptic multiple polylogarithm $I_{\Gamma}(q ; \zeta)$ evaluated at the point $\zeta=1$.

\section{EXAmples of MOdUlar GRAPH FUnCTiOnS}

In this section we shall illustrate the structure of modular graph functions $I_{\Gamma}(q)$ and their associated single-valued elliptic multiple polylogarithms $I_{\Gamma}(q ; \zeta)$ on some simple but significant examples. It will be convenient to characterise the families of graphs under consideration by the number of loops $L$ of the graph $\Gamma$ in the corresponding modular graph function $I_{\Gamma}(q)$.

4.1. General one-loop graphs. The first family of examples is based on modular graph functions $I_{\Gamma}(q)$ for graphs $\Gamma$ with a single loop. The associated single-valued elliptic multiple polylogarithm results from a linear chain graph in which a Green functions are concatenated and integrated over their $a-1$ junction points. Such linear chain graphs are important in their own right, but will also provide natural building blocks for higher graphs that involve linear chains. We introduce the graphic notation given in the figure below

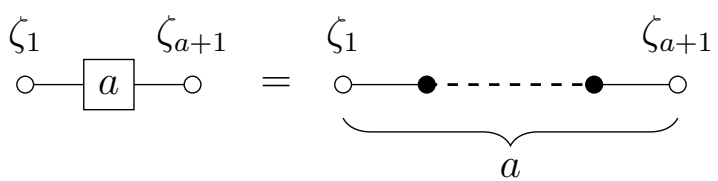

Recall that the black dots correspond to integrated vertices, while the white dots are unintegrated and the corresponding vertices are evaluated at the labels of these points. The weight of the graph is $w=a$, while its number of vertices is $N=a+1$. The associated elliptic function may be evaluated by performing the $a-1$ integrations over the vertices $\zeta_{j}$ for $j=2, \ldots, a$. A convenient way to carry out this evaluation is with the help of the Fourier representation of (2.33). By comparing the result with the Fourier representation given in $(2.33)$ for $D_{a, a}(q ; \zeta)$, we readily find

$$
\prod_{k=2}^{a} \int_{\Sigma} \frac{d^{2} \log \zeta_{k}}{4 \pi^{2} \tau_{2}} \prod_{j=1}^{a} D_{1,1}\left(q ; \zeta_{j+1} / \zeta_{j}\right)=\left(-4 \pi \tau_{2}\right)^{1-a} D_{a, a}\left(q ; \zeta_{a+1} / \zeta_{1}\right)
$$

which is the single-valued elliptic polylogarithm defined in [20]. The single-valuedness in $\zeta=\zeta_{a+1} / \zeta_{1}$ of $D_{a, a}(q ; \zeta)$ is again a consequence of this integral representation, as 
explained in section 3. We may summarise this results by the graphical representation for the linear chain graph in the figure below

$$
a-0=\left(-4 \pi \tau_{2}\right)^{1-a} D_{a, a}\left(q ; \zeta_{a+1} / \zeta_{1}\right) \text {. }
$$

By evaluating the open linear chain graph when the vertices $\zeta_{1}$ and $\zeta_{a+1}$ coincide yields a one-loop chain graph, depicted in the figure below

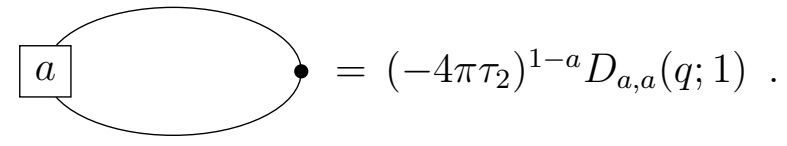

Upon setting $\zeta=1$ in the argument of $D_{a, a}(q ; \zeta)$ in $(2.33)$ we see that

$$
\left(-4 \pi \tau_{2}\right)^{1-a} D_{a, a}(q ; 1)=E_{a}(q)=\sum_{(m, n) \neq(0,0)} \frac{\tau_{2}^{a}}{\pi^{a}|m \tau+n|^{2 a}} .
$$

The representation of the real analytic $E_{a}$ Eisenstein series as a one-loop graph was used in $[9,17,18]$. The Eisenstein series is the special value at $\zeta=1$ of the single-valued elliptic polylogarithm function $D_{a, a}(q ; \zeta)$.

4.2. General two-loop graphs. The general two-loop graph that produces a modular graph function is given by the modular functions $I_{\Gamma}(q)=C_{a, b, c}(q)$ introduced and studied extensively in [9]. ${ }^{6}$ These graphs always have two trivalent vertices, along with $a+b+c-3$ bivalent vertices. For any assignment of value of $a, b, c \geq 1$, a single-valued elliptic multiple polylogarithm $I_{\Gamma}(q ; \zeta)=C_{a, b, c}(q ; \zeta)$ may be constructed from $\Gamma$ by detaching one edge from one of the two trivalent vertices. By permutation symmetry of the indices $a, b, c$, we may detach the edge labeled $a$ without loss of generality, to obtain the graph shown in the figure below

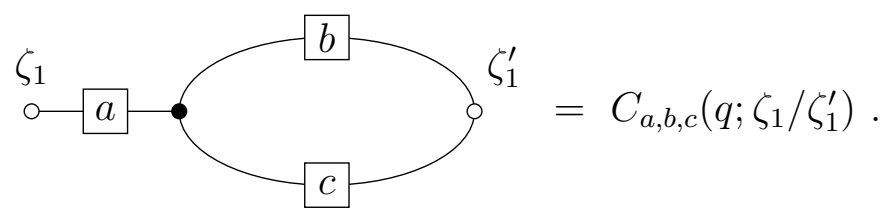

The associated single-valued elliptic multiple polylogarithm, $C_{a, b, c}(q ; \zeta)$ is a function of the ratio $\zeta=\zeta_{1} / \zeta_{1}^{\prime}$ and has the form

$$
C_{a, b, c}(q ; \zeta)=\left(-4 \pi \tau_{2}\right)^{3-a-b-c} \int_{\Sigma} \frac{d^{2} \log \zeta_{2}}{4 \pi^{2} \tau_{2}} D_{a, a}\left(q ; \zeta / \zeta_{2}\right) D_{b, b}\left(q ; \zeta_{2}\right) D_{c, c}\left(q ; \zeta_{2}\right) .
$$

Setting $\zeta=1$ leads to the modular graph function $C_{a, b, c}(q)$

$$
C_{a, b, c}(q)=C_{a, b, c}(q ; 1)
$$

associated with the Feynman graph

\footnotetext{
${ }^{6}$ This is a slight change of notation from [9] where we used $C_{a, b, c}(\tau)$ to represent this function.
} 


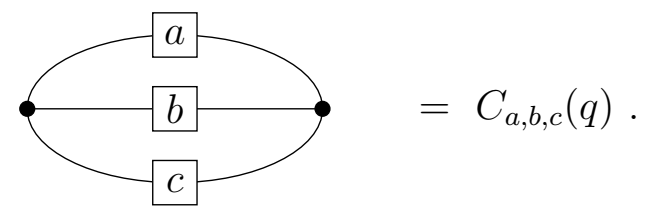

It follows that $C_{a, b, c}(q)$ is the value at $\zeta=1$ of $C_{a, b, c}(q ; \zeta)$, which is a single-valued function of $\zeta$. This illustrates the general point made in section 3.3 and Proposition 3.4.

Using the lattice sum expression for $D_{a, a}(q ; \zeta)$ given in $(2.33)$ leads to the multiple sum representation studied in $[9,10]$

$$
C_{a, b, c}(q)=\sum_{p_{1}, p_{2}, p_{3} \in \mathbb{Z} \tau+\mathbb{Z}}^{\prime}\left(\frac{\tau_{2}}{\pi}\right)^{a+b+c} \frac{\delta\left(p_{1}+p_{2}+p_{3}\right)}{\left|p_{1}\right|^{2 a}\left|p_{2}\right|^{2 b}\left|p_{3}\right|^{2 c}}
$$

where $p_{i}=m_{i} \tau+n_{i}$ parametrises the momenta on the torus $\Sigma$. In [9] linear combinations of the functions $C_{a, b, c}(q)$ were shown to satisfy Laplace eigenvalue equations with source terms that are quadratic in real analytic Eisenstein series. In principle, these equations determine the form of these functions.

When at least one of the indices $a, b, c$ is greater than one, the graph $\Gamma$ contains bivalent vertices in addition to its two trivalent vertices. When this is the case, one may detach the edges meeting at any of the bivalent vertices, to obtain another singlevalued elliptic multiple polylogarithm, specified graphically by the figure below

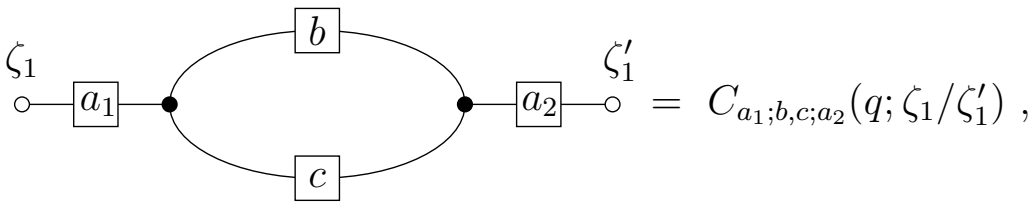

where $a_{1}+a_{2}=a$ and $a_{1}, a_{2} \geq 0$. This case provides the simplest example of a general phenomenon, namely that to a given modular graph function, there will correspond a number of different and generally inequivalent single-valued elliptic multiple polylogarithm functions. Finally, we note that the above modular two-loop graph functions produce, upon detaching both edges on a single trivalent vertex, a star graph with three branches, and thereby a two-variable single-valued elliptic multiple polylogarithm. We shall examine such graphs further in section 5.1 below.

4.3. General three-vertex graphs. The modular graph function associated with an arbitrary graph with a total of three vertices is obtained as a special value of the single-valued elliptic multiple polylogarithm associated with the following graph with two unintegrated vertices

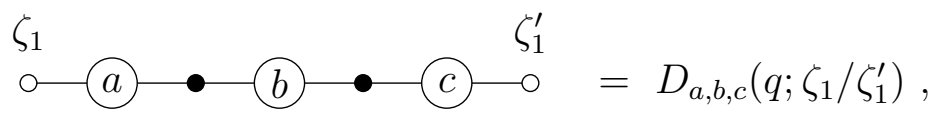


where the encircled numbers indicate the number of the Green functions $D_{1,1}$ which appear in the corresponding link, following our earlier graphical representations. The corresponding single-valued elliptic multiple polylogarithm evaluates as follows

$$
D_{a, b, c}(q ; \zeta)=\prod_{k=2,3} \int_{\Sigma} \frac{d^{2} \log \zeta_{k}}{4 \pi^{2} \tau_{2}} D_{1,1}\left(q ; \zeta / \zeta_{2}\right)^{a} D_{1,1}\left(q ; \zeta_{2} / \zeta_{3}\right)^{b} D_{1,1}\left(q ; \zeta_{3}\right)^{c}
$$

which is a single-valued function of $\zeta$. Its restriction to $\zeta=1$ gives

$$
D_{a, b, c}(q)=D_{a, b, c}(q ; 1),
$$

corresponding to the modular graph function associated with the figure below

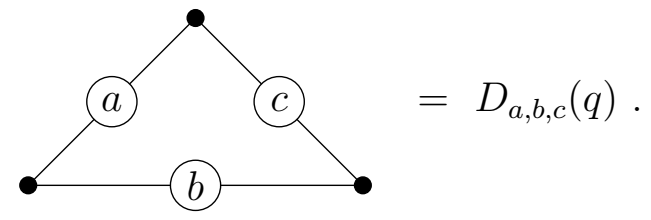

4.4. General graphs. The examples of entire classes of modular graph functions, and their associated single-valued elliptic multiple polylogarithms, provided in the earlier paragraphs lend themselves to a natural generalisation. From a general Feynman graph $\Gamma$, with associated modular graph function $I_{\Gamma}(q)$, it is possible to construct at least one, but in general several inequivalent, single-valued elliptic multiple polylogarithms $I_{\Gamma}(q ; \zeta)$ by detaching one or several Green function edges from any one of the vertices in the graph. The original vertex may be placed at an arbitrary reference point, which we choose to be 1, while the vertex of the detached Green function(s) is placed at an arbitrary point $\zeta$. These observations show the validity of Proposition 3.4 and provide an explicit construction for $I_{\Gamma}(q ; \zeta)$. The significance of the fact that several inequivalent single-valued elliptic multiple polylogarithms arise in this way from a single modular graph function, remains to be explored in full.

\section{Single-VAlued multiple POlylogarithms FOR GRAPhS}

In the preceding two sections, we have introduced modular graph functions, and their relation with single-valued elliptic multiple polylogarithms, leading to Proposition 3.4, and a series of concrete examples. In the present section, we shall make progress towards understanding the description in terms of (non-single-valued) polylogarithms of both modular graph functions and single-valued multiple polylogarithms. In particular, we shall advance a Conjecture as to this general structure, and offer a proof for a certain infinite subclass of graphs, leaving a complete proof for the general case for future investigations. Assuming the validity of our conjecture, we shall infer important properties for the ring structure over $\mathbb{Q}$ of the coefficients of the Laurent series in powers of $\tau_{2}$ of general modular graph functions in terms of single-valued multiple zeta values. The separate terms in the expansions to be discussed later in this section will not be elliptic functions. This is not necessary for the proof of single-valuedness that follows. 
However, the sum of terms is guaranteed to be elliptic since the initial expression for any modular graph function is manifestly elliptic. The Laurent series is the dominant contribution to these modular functions in the limit $\tau_{2} \rightarrow \infty$.

The main conjecture of this paper applies to any single-valued multiple polylogarithm $I_{\Gamma}(q ; \zeta)$ associated with a single-component graph modular graph function $I_{\Gamma}(q)$. By single-component graph, we mean a connected graph, whose associated modular graph function cannot be reduced to the product of lower weight modular graph functions.

Conjecture The single-valued elliptic multiple polylogarithm $I_{\Gamma}(q ; \zeta)$ associated with a single component graph $\Gamma=\Gamma_{w, N}$, with $w$ Green functions, $N+1$ vertices, and $L=w-N$ loops, is a linear combination of multiple polylogarithms of depth at most $L$ and weight at most $w+N-1$.

5.1. Star graphs. We shall now provide a proof of the above Conjecture for the infinite class of star graphs. A graph $S$ is a star graph provided it contains a single integrated $n$-valent vertex with $n>2$, and an arbitrary number of integrated bivalent vertices. By construction, the graph then has $n$ unintegrated end points $\zeta_{i}$ with $i=1, \ldots, n$. To obtain the modular graph function with two $n$-valent vertices associated with $S$ it suffices to set all $\zeta_{i}$ equal to 1 . To obtain single-valued multiple polylogarithms from $S$, one may set one non-empty subset of end points to 1 while setting the remaining non-empty set of endpoints to $\zeta$. A general $n$-valent star graph $S$, with $a_{i}$ Green functions on leg $i$ of the star, along with its multi-variable function value $I_{S}\left(q ; \zeta_{1}, \ldots, \zeta_{n}\right)$, is represented in the figure below

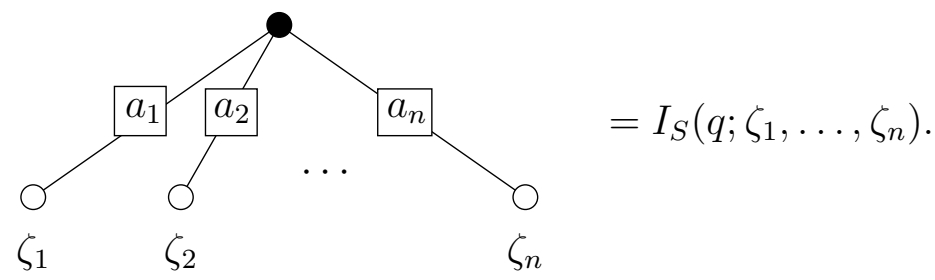

Its associated multi-variable modular graph function evaluates to

$$
I_{S}\left(q ; \zeta_{1}, \ldots, \zeta_{n}\right)=\int_{\Sigma} \frac{d^{2} \log \zeta}{4 \pi^{2} \tau_{2}} \prod_{i=1}^{n} D_{a_{i}, a_{i}}\left(q ; \zeta / \zeta_{i}\right) .
$$

To prove the Conjecture for star graphs, we need to show that the result of performing the integration of the $n$-valent vertex $\zeta$ over the torus $\Sigma$ produces a linear combination, with rational coefficients, of (non-single-valued) multiple polylogarithms, resulting in a formula schematically represented as follows

$$
\sum_{s} \sum_{b_{1}, \ldots, b_{s}} c_{b_{1}, \ldots, b_{s}} \operatorname{Li}_{b_{1}, \ldots, b_{s}}\left(z_{1}, \ldots, z_{s}\right)
$$


where the coefficients $c_{b_{1}, \ldots, b_{s}}$ are rational numbers, the multiple polylogarithm is defined in (2.14), and $z_{i}$ are products of the arguments, generically of the form

$$
q^{m} \bar{q}^{m^{\prime}} \prod_{t} \zeta_{t}^{\alpha_{t}} \bar{\zeta}_{t}^{\beta_{t}}
$$

where $\alpha_{t}, \beta_{t}$ are integers with $1 \leq t \leq s$. We will proceed by considering some algebraic features of the integrand

$$
\prod_{i=1}^{n} D_{a_{i}, a_{i}}\left(q ; \zeta / \zeta_{i}\right)
$$

making use of the fact that the generalised Green functions $D_{a_{i}, a_{i}}\left(q ; \zeta / \zeta_{i}\right)$ are themselves polylogarithms of depth 1 and weight $a_{i}$ given in (2.32) via (2.10), and then perform the integrations over the phase $v$ and the variable $u$ of $\zeta=e^{2 \pi i(u \tau+v)}$. The intermediate steps will not be manifestly single-valued in the $\zeta_{i}$ values but the expression in eq. (5.1) guaranties the single-valueness of the final answer.

5.2. Integral over the phase $v$. We begin by recasting the functions $D_{a_{i}, a_{i}}\left(q ; \zeta / \zeta_{i}\right)$ in terms of the functions $D_{a_{i}, a_{i}}\left(q^{m}\left(\zeta / \zeta_{i}\right)^{ \pm 1}\right)$ and their complex conjugate with the help of equation (2.36), and then expressing the latter as a linear combination of polylogarithms $\mathrm{Li}_{k}$ using (2.10). In performing the $v$-integral, the presence of the Bernoulli polynomials $B_{2 a}(u)$ in $(2.36)$ will be immaterial, as will be the logarithms in (2.10), since they involve $u$ but do not depend on $v$. We will suppress their presence in this section. Finally, the arguments entering the polylogarithms $\operatorname{Li}_{k}$ in $D_{a_{i}, a_{i}}$ depend on $\zeta$ either through a factor linear in $\zeta$, or a factor linear in $\zeta^{-1}$. It will be convenient in the sequel to group together the factors in $\zeta$ and those in $\zeta^{-1}$, of which we shall assume there are respectively $r$ and $s$. The maximal values of these parameters are characterised by $0 \leq r, s$ and $r+s \leq n$. Thus, the $v$-integrals required to evaluate $I_{S}$ take the form

$$
J_{\mathbf{a}, \mathbf{b}}(\mathbf{x}, \mathbf{y})=\int_{0}^{1} d v \prod_{i=1}^{r} \operatorname{Li}_{a_{i}}\left(x_{i} e^{2 \pi i v}\right) \prod_{j=1}^{s} \operatorname{Li}_{b_{j}}\left(y_{j} e^{-2 \pi i v}\right),
$$

where we have introduced the following notation,

$$
\begin{array}{ll}
\mathbf{a}=\left(a_{1}, \ldots, a_{r}\right) & \mathbf{x}=\left(x_{1}, \ldots, x_{r}\right) \\
\mathbf{b}=\left(b_{1}, \ldots, b_{s}\right) & \mathbf{y}=\left(y_{1}, \ldots, y_{s}\right) .
\end{array}
$$

The entries of the composite indices $\mathbf{a}$ and $\mathbf{b}$ are positive integers, while the entries of $\mathbf{x}$ and $\mathbf{y}$ depend on $u$, and on positive powers of $q$ and positive powers of $\bar{q}$, but are independent of $v$. The variables obey $\left|x_{i}\right|,\left|y_{j}\right|<1$ for all $i=1, \ldots, r$ and $j=1, \ldots, s$. In view of these bounds, we may use the series representations for the functions Li to perform the integration over $v$ which results in the insertion into the sum of a Kronecker 
$\delta$-function,

$$
J_{\mathbf{a}, \mathbf{b}}(\mathbf{x}, \mathbf{y})=\sum_{0<m_{1}, \ldots, m_{r}} \sum_{0<n_{1}, \ldots, n_{s}} \delta\left(\sum_{i=1}^{r} m_{i}-\sum_{j=1}^{s} n_{j}\right) \prod_{i=1}^{r} \frac{x_{i}^{m_{i}}}{m_{i}^{a_{i}}} \prod_{j=1}^{s} \frac{y_{j}^{n_{j}}}{n_{j}^{b_{j}}} .
$$

To prove that this constrained sum may be expressed as a linear combination, with rational coefficients, of functions $\mathrm{Li}$ evaluated on various combinations of the variables $\mathbf{x}, \mathbf{y}$, we shall proceed by induction on $s$, at fixed but arbitrary value of $r$. For $r>0$, and $s=0$, the integral vanishes identically.

To initiate the induction, consider first the case $s=1$. It is straightforward to solve for $n_{1}$ which is given by $n_{1}=m_{1}+\cdots+m_{r}$. The constraint $n_{1}>0$ imposed by the definition of $J_{\mathbf{a}, \mathbf{b}}$ is automatically satisfied since all the integers $m_{i}$ are strictly positive. Thus, we find

$$
J_{\mathbf{a}, b_{1}}\left(\mathbf{x}, y_{1}\right)=\sum_{0<m_{1}, \ldots, m_{r}} \frac{1}{m_{1}+\cdots+m_{r}} \prod_{i=1}^{r} \frac{\left(x_{i} y_{1}\right)^{m_{i}}}{m_{i}^{a_{i}}} .
$$

The summation may be expressed equivalently as the following integral

$$
J_{\mathbf{a}, b_{1}}\left(\mathbf{x}, y_{1}\right)=\int_{0}^{1} \frac{d t}{t} \prod_{i=1}^{r} \operatorname{Li}_{a_{i}}\left(x_{i} y_{1} t\right) .
$$

Using the stuffle relations of (2.17), the product over the Li-functions may be expressed as a linear combination, with rational coefficients, of multiple polylogarithms. The one of highest depth $r$ is given by $\operatorname{Li}_{a_{1}, \ldots, a_{r}}\left(x_{1} y_{1} t, \ldots, x_{r} y_{1} t\right)$, while the one of lowest depth 1 is given by $\operatorname{Li}_{a_{1}+\cdots+a_{r}}\left(x_{1} \ldots x_{r} y_{1}^{r} t^{r}\right)$, along with all stuffle combinations with rational coefficients and depths in between 1 and $r$. The integral over $t$ of any of these Lifunctions gives back an Li-function of various combinations of the arguments. This concludes the proof of the claim for $s=1$.

Next, we shall assume that the claimed decomposition property of $J_{\mathbf{a}, \mathbf{b}}$ defined in (5.5) holds true for all indices $\mathbf{a}, \mathbf{b}$ for any value of $r, s$. We now wish to prove by induction that the decomposition property will then also hold for all indices $\mathbf{a}, \mathbf{b}^{\prime}$ for all $r$, and $s$ replaced by $s+1$. The corresponding $J$-function is given by

$$
J_{\mathbf{a}, \mathbf{b}^{\prime}}\left(\mathbf{x}, \mathbf{y}^{\prime}\right)=\sum_{\substack{0<m_{1}, \ldots, m_{r} \\ 0<n_{1}, \ldots, n_{s}, n_{s+1}}} \delta\left(\sum_{i=1}^{r} m_{i}-\sum_{j=1}^{s} n_{j}-n_{s+1}\right) \prod_{i=1}^{r} \frac{x_{i}^{m_{i}}}{m_{i}^{a_{i}}} \prod_{j=1}^{s+1} \frac{y_{j}^{n_{j}}}{n_{j}^{b_{j}}} .
$$

and we use the notation $\mathbf{b}^{\prime}=\left(b_{1}, \ldots, b_{s}, b_{s+1}\right)$ and $\mathbf{y}^{\prime}=\left(y_{1}, \ldots, y_{s}, y_{s+1}\right)$.

To solve the Kronecker $\delta$-function constraint now requires more care than in the case $s=1$. One cannot simply use the solution $n_{s+1}=m_{1}+\ldots m_{r}-\left(n_{1}+\cdots+n_{s}\right)$, since then $n_{s+1}$ is not guaranteed to obey the constraint that it must be positive. Therefore, we shall proceed as follows instead. We single out an arbitrary $m$-variable, say $m_{1}$, and compare the values of $n_{s+1}$ allowed by the $\delta$-function constraint to the value of 
$m_{1}$. We have three cases, namely $n_{s+1}=m_{1}, n_{s+1}<m_{1}$ or $n_{s+1}>m_{1}$, and split the sum which defines the function $J$ accordingly into three terms

$$
J_{\mathbf{a}, \mathbf{b}^{\prime}}\left(\mathbf{x}, \mathbf{y}^{\prime}\right)=J^{0}+J^{+}+J^{-}
$$

where the terms $J^{0}, J^{+}$, and $J^{-}$respectively include the contributions to the sum from $n_{s+1}=m_{1}, n_{s+1}<m_{1}$, and $n_{s+1}>m_{1}$.

For the contributions to the sum with $n_{s+1}<m_{1}$, which are regrouped in $J^{+}$, we parametrise the difference by $m_{1}=n_{s+1}+k$ with $k$ ranging over all positive integers. The sum becomes,

$$
J^{+}=\sum_{\substack{0<k, m_{2}, \ldots, m_{r} \\ 0<n_{1}, \ldots, n_{s}, n_{s+1}}} \delta\left(k+\sum_{i=2}^{r} m_{i}-\sum_{j=1}^{s} n_{j}\right) \frac{x_{1}^{k+n_{s+1}}}{\left(k+n_{s+1}\right)^{a_{1}}} \prod_{i=2}^{r} \frac{x_{i}^{m_{i}}}{m_{i}^{a_{i}}} \prod_{j=1}^{s+1} \frac{y_{j}^{n_{j}}}{n_{j}^{b_{j}}}
$$

We re-baptise the variable $k$ as $m_{1}$, setting $k=m_{1}$ in the sum. As a result, we have

$$
J^{+}=\sum_{\substack{0<m_{1}, \ldots, m_{r} \\ 0<n_{1}, \ldots, n_{s}, n_{s+1}}} \delta\left(\sum_{i=1}^{r} m_{i}-\sum_{j=1}^{s} n_{j}\right) \frac{x_{1}^{m_{1}+n_{s+1}}}{\left(m_{1}+n_{s+1}\right)^{a_{1}}} \prod_{i=2}^{r} \frac{x_{i}^{m_{i}}}{m_{i}^{a_{i}}} \prod_{j=1}^{s+1} \frac{y_{j}^{n_{j}}}{n_{j}^{b_{j}}}
$$

Note that the $\delta$-function constraint involves only the first $r+s$ summation variables but does not involve $n_{s+1}$. This observation is crucial to make the inductive proof possible. The summations over $m_{1}$ and $n_{s+1}$ are coupled only through the denominator factor $\left(m_{1}+n_{s+1}\right)^{-a_{1}}$ but not through the Kronecker $\delta$-function constraint. To complete the inductive argument, we decouple the summations over $m_{1}$ and $n_{s+1}$ by differentiating in $x_{1}$. The independent sums are then easily regrouped, and lead to the following differential equation

$$
\left(x_{1} \frac{\partial}{\partial x_{1}}\right)^{a_{1}} J^{+}=\operatorname{Li}_{b_{s+1}}\left(x_{1} y_{s+1}\right) \operatorname{Li}_{0, a_{2}, \ldots, a_{r}, b_{1}, \ldots, b_{s}}\left(x_{1}, \ldots, x_{r}, y_{1}, \ldots, y_{s}\right)
$$

Stuffle identities (2.17) may again be used to decompose this product into a linear combination, with rational coefficients, of Li-functions of depth $r+s+1$. The above differential equation for $J^{+}$may then be integrated iteratively using (2.15) in terms of linear combinations of Li-functions of depth $r+s+1$. This concludes the part of the proof for the contributions $J^{+}$to $J_{\mathbf{a}, \mathbf{b}}(\mathbf{x}, \mathbf{y})$.

For the contributions to the sum with $n_{s+1}>m_{1}$, which are regrouped in $J^{-}$, we parametrise the difference by $m_{1}+k=n_{s+1}$ with $k$ ranging over all positive integers. The arguments now proceed as in the case of $J^{+}$with labels of positive and negative powers of $\zeta$ reversed. 
Finally, for the contributions to the sum with $n_{s+1}=m_{1}$, which are regrouped in $J^{0}$, we have

$$
J^{0}=\sum_{\substack{0<m_{1}, \ldots, m_{r} \\ 0<n_{1}, \ldots, n_{s}, n_{s+1}}} \delta\left(\sum_{i=2}^{r} m_{i}-\sum_{j=1}^{s} n_{j}\right) \frac{\left(x_{1} y_{s+1}\right)^{m_{1}}}{m_{1}^{a_{1}+b_{s+1}}} \prod_{i=1}^{r} \frac{x_{i}^{m_{i}}}{m_{i}^{a_{i}}} \prod_{j=1}^{s} \frac{y_{j}^{n_{j}}}{n_{j}^{b_{j}}} .
$$

The sum over $m_{1}$ is now completely decoupled from the other sums, and we have

$$
J^{0}=\operatorname{Li}_{a_{1}+b_{s+1}}\left(x_{1} y_{s+1}\right) \operatorname{Li}_{a_{2}, \ldots, a_{r}, b_{1}, \ldots, b_{s}}\left(x_{2}, \ldots, x_{r}, y_{1}, \ldots, y_{s}\right)
$$

Applying the stuffle relations of (2.17) allows us to decompose this product again into a linear combination with rational coefficients of Li-functions. This completes the proof for all contributions to $J_{\mathbf{a}, \mathbf{b}}(\mathbf{x}, \mathbf{y})$, and thus of this function itself.

5.3. Arguments for the $v$-integral in the general case. While the proof of the Conjecture given in the preceding section is complete for star graphs, and graphs derived from it, we need further results to support the Conjecture for general graphs. In this subsection, our aim will not be to provide a complete proof for general graphs, but rather to show that some of the key novel results that are needed for general graphs but were not required for star graphs, can be established in a satisfactory way.

One formulation may be obtained by considering the $v$-integral of a general multiple polylogarithm, but with arguments given by higher powers of $\zeta$ and $\zeta^{-1}$ (for star graphs only arguments linear in $\zeta$ and $\zeta^{-1}$ were required). This will lead to a general $v$-integral of the form

$$
\mathcal{J}_{\mathbf{a}}^{\gamma}(\mathbf{z})=\int_{0}^{1} d v \operatorname{Li}_{\mathbf{a}}\left(z_{1} e^{2 \pi i \gamma_{1} v}, \ldots, z_{r} e^{2 \pi i \gamma_{r} v}\right)
$$

where the exponent $\gamma=\left(\gamma_{1}, \ldots, \gamma_{r}\right)$ has integer entries, while $\mathbf{a}=\left(a_{1}, \ldots, a_{r}\right)$ has positive integer entries, and the arguments are again bounded by $\left|z_{i}\right|<1$ for all $i=1, \ldots, r$. By expanding the Li-function in a power series in $z_{i}$, we may readily perform the integral over $v$ to obtain

$$
\mathcal{J}_{\mathbf{a}}^{\gamma}(\mathbf{z})=\sum_{0<\mu_{1}<\cdots<\mu_{r}} \delta\left(\sum_{i=1}^{r} \gamma_{i} \mu_{i}\right) \prod_{i=1}^{r} \frac{z_{i}^{\mu_{i}}}{\mu_{i}^{a_{i}}}
$$

where the summation is over ordered positive integers $\mu_{1}, \ldots, \mu_{r}$. The case considered earlier corresponds to having $\gamma_{i}= \pm 1$, so that the Kronecker $\delta$-function constraint may be solved to provide an iterative proof as we did in the preceding subsection. When $\gamma_{i} \neq \pm 1$, however, one needs to deal with the issues of divisibility in solving the constraint equation imposed by the Kronecker $\delta$-function.

We begin by parametrising the ordering conditions on the indices $\mu_{i}$ by setting $\mu_{0}=0$ and introducing the following change of variables

$$
\mu_{i}=\mu_{i-1}+m_{i} \quad 0<m_{i} \quad 1 \leq i \leq r .
$$


The variables $m_{i}$ are now constrained only to be positive, but no further ordering requirement between them is being imposed. Importantly, this change of variables has the effect of decoupling the ordering conditions between different $m_{i}$. Next, it will be convenient to express $\gamma$ and $\mathbf{z}$ in this new basis and we define

$$
\alpha_{i}=\sum_{j=i}^{r} \gamma_{j} \quad x_{i}=\prod_{j=i}^{r} z_{j} .
$$

As a result, the function $\mathcal{J}_{\mathbf{a}}^{\gamma}(\mathbf{z})$ admits the following sum representation

$$
\mathcal{J}_{\mathbf{a}}^{\gamma}(\mathbf{z})=\sum_{0<m_{1}, \ldots, m_{r}} \delta\left(\sum_{i=1}^{r} \alpha_{i} m_{i}\right) \prod_{i=1}^{r} \frac{x_{i}^{m_{i}}}{\mu_{i}^{a_{i}}} .
$$

To save unnecessary extra notation, the denominator factors have been left in terms of $\mu_{i}$ and should be viewed as given in terms of the functions of the $m_{i}$ by (5.19). Without loss of generality, we shall assume that all $\alpha_{i}$ are non-vanishing; in the contrary case, the corresponding summation indices do not enter into the Kronecker $\delta$-function constraint and the sum reduces to a case with lower value of $r$.

Next, we partition the set of indices $\{1,2, \ldots, r\}$ into two sets $I_{+}$and $I_{-}$according to whether $\alpha_{i}$ is positive or negative

$$
\begin{aligned}
& \alpha_{i}>0 \quad \Longrightarrow \quad i \in I_{+} \\
& \alpha_{i}<0 \quad \Longrightarrow \quad i \in I_{-} .
\end{aligned}
$$

It will be convenient to work with positive exponents only, so we shall set $\beta_{i}=-\alpha_{i}$ whenever $i \in I_{-}$, so that $\beta_{i}>0$. The summation may then be rearranged as follows

$$
\mathcal{J}_{\mathbf{a}}^{\gamma}(\mathbf{z})=\sum_{0<m_{1}, \ldots, m_{r}} \delta\left(\sum_{i \in I_{+}} \alpha_{i} m_{i}-\sum_{i \in I_{-}} \beta_{i} m_{i}\right) \prod_{i=1}^{r} \frac{x_{i}^{m_{i}}}{\mu_{i}^{a_{i}}} .
$$

We now proceed iteratively on the value of $n$, and assume that the extended array $\gamma^{\prime}=\left(\gamma_{1}, \ldots, \gamma_{r}, \gamma_{r+1}\right)$ produces one additional entry belonging to $I_{-}$, which corresponds to an additional exponent $\beta_{i_{-}}>0$ and an additional summation variable $m_{i_{-}}>0$.

We now wish to reduce the Kronecker $\delta$-function involving $n+1$ summation variables $m$ to one that involves only $n$ summation variables, imitating the procedure we followed for star graphs. To this end, we single out an index $i_{+}$in $I_{+}$corresponding to $m_{i_{+}} \alpha_{i_{+}}$. Thus, we need to reduce the following constraint on $n+1$ summation variables $m_{1}, \ldots, m_{n+1}$

$$
\sum_{i \in I_{+} \backslash\left\{i_{+}\right\}} \alpha_{i} m_{i}-\sum_{i \in I_{-}} \alpha_{i} m_{i}+\alpha_{i_{+}} m_{i_{+}}-\beta_{i_{-}} m_{i_{-}}=0
$$

to a summation involving only $n$ summation variables. We proceed as in the case of star graphs, but take careful account of the divisibility issues. We shall distinguish contributions for which $\alpha_{i_{+}} m_{i_{+}}=\beta_{i_{-}} m_{i_{-}}$from those for which $\alpha_{i_{+}} m_{i_{+}}>\beta_{i_{-}} m_{i_{-}}$and 
those for which $\alpha_{i_{+}} m_{i_{+}}<\beta_{i_{-}} m_{i_{-}}$. We shall refer to these partial sums respectively as $\mathcal{J}^{0}, \mathcal{J}^{+}$, and $\mathcal{J}^{-}$.

We shall concentrate here on the contribution $\mathcal{J}^{+}$, arising from the contributions satisfying $\alpha_{i_{+}} m_{i_{+}}>\beta_{i_{-}} m_{i_{-}}$. The other cases are analogous, following the model of the star graphs. The integers $\alpha_{i_{+}}$and $\beta_{i_{-}}$are both positive, and have been assumed to be non-zero. We shall denote their greatest common divisor by $\alpha_{\star}>0$. There now exists a unique solution $m_{i_{ \pm}}^{0}$ to the Bézout equation

$$
\alpha_{i_{+}} m_{i_{+}}^{0}-\beta_{i_{-}} m_{i_{-}}^{0}=\alpha_{\star}
$$

with the properties $0 \leq m_{i_{+}}^{0}<\beta_{i_{-}} / \alpha_{\star}$ and $0 \leq m_{i_{-}}^{0}<\alpha_{i_{+}} / \alpha_{\star}$. Note that this solution is determined completely by the nature of $\alpha_{i_{+}}$and $\beta_{i_{-}}$and does not in any way involve the summation variables $m$. Having determined $m_{i_{ \pm}}^{0}$, we may now recast the constraint as follows

$$
\sum_{i \in I_{+} \backslash\left\{i_{+}\right\}} \alpha_{i} m_{i}-\sum_{i \in I_{-}} \alpha_{i} m_{i}+\alpha_{\star} k=0
$$

for an integer $k$ which is positive by the assumption $\alpha_{i_{+}} m_{i_{+}}>\beta_{i_{-}} m_{i_{-}}$. As a result, the Kronecker $\delta$-function constraint now involves only $n$ summation variables. The complete parametrisation of the summation variables $m_{i_{ \pm}}$is given in terms of the integer $k>0$, and an additional integer summation variable $\ell$, as follows

$$
\begin{aligned}
& m_{i_{+}}=k m_{i_{+}}^{0}+\ell \frac{\beta_{i_{-}}}{\alpha_{\star}} \\
& m_{i_{-}}=k m_{i_{-}}^{0}+\ell \frac{\alpha_{i_{+}}}{\alpha_{\star}}
\end{aligned}
$$

The variable $\ell$ does not enter into the constraint. In general, the range of $\ell$ will include both positive, zero, and negative integers, and must itself be subdivided into these three sub-ranges. Within each sub-range, the summation over $\ell$ may be performed, after sufficient derivatives in the external variables $x_{i}$ have been taken to deal with the denominator factors $\mu_{i}^{a_{i}}$, in a manner analogous to how we proceeded for star graphs. This concludes our discussion of the arguments needed to prove that the integral over $v$ produces a linear combination, with rational coefficients, of Li-functions.

5.4. Integral over the variable $u$. The second type of integral involves integration over one of the variables $u_{i}$, leading to the integrals of the type

$$
\mathcal{K}_{a, \mathbf{a}}^{\alpha}(\mathbf{x})=\int_{0}^{1} d u u^{a} \operatorname{Li}_{a_{1}, \ldots, a_{r}}\left(x_{1} e^{-2 \pi \tau_{2} \alpha_{1} u}, \ldots, x_{r} e^{-2 \pi \tau_{2} \alpha_{r} u}\right)
$$

where the variables $x_{i}$ are independent of $u$ and bounded by $\left|x_{i}\right|<1$, while $a, a_{i}$ and $\alpha_{i}$ are positive or zero integers for all $i=1, \ldots, r$. We have introduced the notation $\alpha=$ $\left(\alpha_{1}, \ldots, \alpha_{r}\right)$. Actually, the integrals make perfect sense when $\alpha_{i}$ is a positive integer, and it will sometimes be convenient for performing intermediate calculations, to let $\alpha_{i}$ be real. The multiple polylogarithm arises from stuffle relations in the expansion of 
products of Green functions on the torus, while the pre-factor $u^{a}$ arises from expanding the Bernoulli polynomials in $D_{a_{i}, a_{i}}(\zeta)$.

It is sufficient to study the integrals $\mathcal{K}_{0, \mathbf{a}}^{\alpha}$, since cases with $a>0$ may be obtained by differentiating with respect to one the arguments $\alpha_{i}$ (temporarily allowing it to be a real variable instead of an integer) of the polylogarithms using the differentiation rule of the multiple polylogarithms in (2.15). Using the sum representation of the multiple polylogarithms and the integral

$$
\int_{0}^{1} d u e^{-2 \pi \tau_{2} u \alpha}=\frac{1}{2 \pi \tau_{2}} \frac{1}{\alpha}\left(1-e^{-2 \pi \tau_{2} \alpha}\right)
$$

gives

$$
\mathcal{K}_{0, \mathbf{a}}^{\alpha}(\mathbf{x})=\frac{1}{2 \pi \tau_{2}} \sum_{0<m_{1}<\cdots<m_{r}} \prod_{i=1}^{r} \frac{x_{i}^{m_{i}}}{m_{i}^{a_{i}}} \frac{1-e^{-2 \pi \tau_{2} \sum_{i=1}^{r} \alpha_{i} m_{i}}}{\sum_{i=1}^{r} \alpha_{i} m_{i}}
$$

By differentiation we obtain

$$
\begin{aligned}
\left(\sum_{i=1}^{r} \alpha_{i} x_{i} \frac{d}{d x_{i}}\right) \mathcal{K}_{0, \mathbf{a}}^{\alpha}(\mathbf{x})=\frac{1}{2 \pi \tau_{2}}\left(\operatorname{Li}_{a_{1}, \ldots, a_{r}}\left(x_{1}, \ldots, x_{r}\right)\right. & \\
& \left.\quad-\operatorname{Li}_{a_{1}, \ldots, a_{r}}\left(x_{1} e^{-2 \pi \tau_{2} \alpha_{1}}, \ldots, x_{r} e^{-2 \pi \tau_{2} \alpha_{r}}\right)\right) .
\end{aligned}
$$

Again, using the differential relations of multiple polylogarithms (2.15) one concludes that $\mathcal{K}_{0, \mathbf{a}}^{\alpha}$ is a combination of multiple polylogarithm of depth $r$ and weight $\sum_{i=1}^{r} a_{i}+1$. The same argument implies that $\mathcal{K}_{a, \mathbf{a}}^{\alpha}$ is a linear combination of multiple polylogarithms of depth $r$ and weight $\sum_{i=1}^{r} a_{i}+a$.

\section{Fourier COEFficients of MOdular GRAPH FunCtions}

Having proved the Conjecture for the case of star graphs, and graphs derived from star graphs, and presented an outline of the evidence for the validity of the Conjecture for general graphs, we now proceed to discuss some implications of the Conjecture, assuming it holds true for general graphs.

6.1. Implications for the constant Fourier mode. Assuming the validity of the conjecture implies the following corollary of the conjecture concerning the constant Fourier mode of the Feynman integral $I_{\Gamma}(q ; 1)=I_{\Gamma}(q)$.

\section{Proposition}

Let $I_{\Gamma}^{0}\left(\tau_{2}\right)$ denote the constant Fourier mode of the modular graph function $I_{\Gamma}(q)$ associated with a graph $\Gamma=\Gamma_{w, N}$ with $w$ Green functions and $N$ vertices

$$
I_{\Gamma}^{0}\left(\tau_{2}\right)=\int_{-\frac{1}{2}}^{\frac{1}{2}} d \tau_{1} I_{\Gamma}(q)
$$


(1) The constant Fourier mode $I_{\Gamma}^{0}\left(\tau_{2}\right)$ has an expansion for large $\tau_{2}$ consisting of a Laurent polynomial with a term of highest degree $\left(\pi \tau_{2}\right)^{w}$ and a term of lowest degree $\left(\pi \tau_{2}\right)^{1-w}$ with exponentially suppressed corrections of order $\mathcal{O}\left(e^{-2 \pi \tau_{2}}\right)$.

(2) The coefficients of the $\left(\pi \tau_{2}\right)^{k}$ term $(w \leq k \leq w-1)$ in the Laurent polynomial is a single-valued multiple zeta value of weight $w-k$.

The first item is straightforward to establish directly from the definition of the modular graph functions. The second item follows from the Conjecture since the modular graph function $I_{\Gamma}(q)$ results from evaluating a linear combination, with rational coefficients, of multiple polylogarithms. The coefficient are single-valued multiple zetas as a consequence of the single-valueness with respect to $\zeta$ of the modular graph function $I_{\Gamma}(q, \zeta)$ in eq. (3.14). Since single-valued multiple polylogarithms evaluated at unit argument yield single-valued multiple zeta functions, the validity of the Conjecture supports the validity of item (2) above. Furthermore, item (1) also follows from item (2) since there are no single-valued multiple zeta values of negative weights. By convention a single-valued multiple zeta of weight zero is a rational number.

We shall now provide examples of graphs, and their associated modular graph functions, for which these Laurent polynomials of the constant Fourier mode have been calculated, or for which the general structure of the corresponding Laurent polynomial is understood on general grounds.

6.2. The Eisenstein series. The simplest case where the implications of the Corollary on the constant Fourier mode may be easily verified is the non-holomorphic Eisenstein series itself. The constant Fourier mode consists of a Laurent polynomial in $\tau_{2}$ with just two terms. Throughout, it will be convenient to give the Laurent series in powers of $y=\pi \tau_{2}$ instead of $\tau_{2}$, given the structure of the coefficients announced in item (3) of the implications. For the Eisenstein series, we have

$$
\int_{-\frac{1}{2}}^{\frac{1}{2}} d \tau_{1} E_{a}(q)=(-1)^{a-1} \frac{B_{2 a}}{(2 a) !}(4 y)^{a}+\frac{4(2 a-3) !}{(a-2) !(a-1) !} \zeta(2 a-1)(4 y)^{1-a} .
$$

The coefficients of powers of $y$ are rational numbers multiplying odd $\zeta$-values which are single-valued according the definition used in [6-8]. The leading coefficient is a rational number involving the Bernoulli number $B_{2 a}$, which may also be described as "single-valued" since the Bernoulli polynomials in (2.4) are single-valued. This exemplifies the relation between the world-sheet graphs and the single-valued elliptic multiple polylogarithms in the very simplest cases.

6.3. The $C_{a, b, c}(q)$ modular graph functions. Although explicit expressions for the Laurent series of the general two-loop modular graph $C_{a, b, c}$ are not available in closed form, it is not difficult to verify the compatibility of their structure with the Corollary given above. First of all, the Laurent series of every specific $C_{a, b, c}$ function evaluated explicitly in [9] is of the form predicted by the Corollary, with the highest power being 
$y^{w}$ and the lowest $y^{1-w}$. The simplest case is $C_{1,1,1}=E_{3}+\zeta(3)$, which is manifestly compatible, in view of the Fourier mode expansion of the Eisenstein series given in the preceding section.

The cases of weight 4 and 5 worked out in [9] give the following Laurent expansions

$$
\begin{aligned}
& \int_{-\frac{1}{2}}^{\frac{1}{2}} d \tau_{1} C_{2,1,1}(q)=\frac{2 y^{4}}{14175}+\frac{\zeta(3) y}{45}+\frac{5 \zeta(5)}{12 y}-\frac{\zeta(3)^{2}}{4 y^{2}}+\frac{9 \zeta(7)}{16 y^{3}}+\mathcal{O}\left(e^{-2 y}\right) \\
& \int_{-\frac{1}{2}}^{\frac{1}{2}} d \tau_{1} C_{3,1,1}(q)=\frac{2 y^{5}}{155925}+\frac{2 \zeta(3) y^{2}}{945}-\frac{\zeta(5)}{180}+\frac{7 \zeta(7)}{16 y^{2}}-\frac{\zeta(3) \zeta(5)}{2 y^{3}}+\frac{43 \zeta(9)}{64 y^{4}}+\mathcal{O}\left(e^{-2 y}\right)
\end{aligned}
$$

and these expressions are again compatible with the structure predicted by the Corollary since the coefficients only involve polynomials in odd $\zeta$ values, which are singlevalued multiple zetas.

This structure of the Laurent series is easily seen to follow from the structure of nested differential equations satisfied by the $C_{a, b, c}(q)$ functions. Indeed, every Laplaceeigenvalue equation of [9] for linear combinations (with rational coefficients) $\mathfrak{C}_{w, s, \mathfrak{p}}$ of $C_{a, b, c}$-functions of weight $w=a+b+c$ and eigenvalue $s=1,2, \ldots, w-2$, has an inhomogeneous part consisting of a term linear in the Eisenstein series $E_{w}$ multiplied by a rational number plus terms of the form $E_{w_{1}} E_{w_{2}}$ with $w_{1}, w_{2} \geq 2$ and $w=w_{1}+w_{2}$, and multiplied by rational coefficients. Therefore, the inhomogeneous part involves a Laurent polynomial with powers $y^{k}$ ranging from $1-w \leq k \leq w$, the coefficient of the term $y^{w}$ being rational, and the remaining coefficients being odd $\zeta$-values, which by definition are proportional to single-valued zeta values with rational coefficients. We may represent this by

$$
\left(y^{2} \frac{\partial^{2}}{\partial y^{2}}-s(s-1)\right) \mathfrak{C}_{w, s, \mathfrak{p}} \in \mathbb{Q} E_{w}+\sum_{\substack{w_{1}, w_{2} \geq 2 \\ w_{1}+w_{2}=w}} \mathbb{Q} E_{w_{1}} E_{w_{2}}
$$

The operator on the left hand side of the equation has a kernel consisting of the Laurent terms proportional to $y^{s}$ and $y^{1-s}$. The Laplace eigenvalue equations therefore imply that almost all the coefficients in the Laurent polynomial for $\mathfrak{C}_{w ; s ; \mathfrak{p}}$, except for the highest coefficient of $y^{w}$, are odd zeta values or products thereof, as indeed predicted by the Corollary.

6.4. Examples of higher cases. Some Laurent expansions of modular graph functions that do not belong to the family of graphs $C_{a, b, c}$ were also evaluated in [9]. Here, 
we give just two examples of Laurent series at weight 5 , with $y=\pi \tau_{2}$

$$
\begin{aligned}
& \int_{-\frac{1}{2}}^{\frac{1}{2}} d \tau_{1} D_{3,1,1}(q)=\frac{2 y^{5}}{22275}+\frac{y^{2} \zeta(3)}{45}+\frac{11 \zeta(5)}{60}+\frac{105 \zeta(7)}{32 y^{2}}-\frac{3 \zeta(3) \zeta(5)}{2 y^{3}}+\frac{81 \zeta(9)}{64 y^{4}}+\mathcal{O}\left(e^{-2 y}\right) \\
& \int_{-\frac{1}{2}}^{\frac{1}{2}} d \tau_{1} D_{2,2,1}(q)=\frac{8 y^{5}}{467775}+\frac{4 \zeta(3) y^{2}}{945}+\frac{13 \zeta(5)}{45}+\frac{7 \zeta(7)}{8 y^{2}}-\frac{\zeta(3) \zeta(5)}{y^{3}}+\frac{9 \zeta(9)}{8 y^{4}}+\mathcal{O}\left(e^{-2 y}\right) .
\end{aligned}
$$

In each case, only odd zeta values, or products thereof, occur all of which are singlevalued multi-zeta values. The preceding expressions do not involve non-trivial singlevalued multiple zetas (i.e. ones which are not reducible to polynomials in odd zeta values) since these only arise at weights $\geq 11$.

Explicit computations of Laurent series at higher weight have been performed recently by Zerbini [43]. These strikingly confirm the conjectured structure of the coefficients of the Laurent polynomials. For example, Zerbini's evaluation of the constant Fourier mode of $D_{1,1,5}(q)$ (which is a special case of the class of star graphs) is given by

$$
\begin{aligned}
\frac{1}{4^{7}} \int_{-\frac{1}{2}}^{\frac{1}{2}} d \tau_{1} D_{1,1,5}(q) & =\frac{62 y^{7}}{10945935}+\frac{\zeta_{s v}(3)}{243} y^{4}+\frac{119}{648} \zeta_{s v}(5) y^{2}+\frac{11}{54} \zeta_{s v}(3)^{2} y \\
+ & \frac{21}{32} \zeta_{s v}(7)+\frac{23}{\frac{\zeta_{s v}(3) \zeta_{s v}(5)}{6 y}+\frac{7115 \zeta_{s v}(9)-900 \zeta_{s v}(3)^{3}}{576 y^{2}}} \\
+\frac{1245 \zeta_{s v}(3) \zeta_{s v}(7)-150 \zeta_{s v}(5)^{2}}{64 y^{3}} & \\
+ & \frac{288 \zeta_{s v}(3,5,3)-4080 \zeta_{s v}(5) \zeta_{s v}(3)^{2}-9573 \zeta_{s v}(11)}{256 y^{4}} \\
+ & \frac{2475 \zeta_{s v}(5) \zeta_{s v}(7)+1125 \zeta_{s v}(9) \zeta_{s v}(3)}{128 y^{5}}-\frac{1575}{64} \frac{\zeta_{s v}(13)}{y^{6}}+\mathcal{O}\left(e^{-2 y}\right)
\end{aligned}
$$

The fact that the coefficient of $y^{-4}$ is a linear combination of depth 3 single-valued multiple zeta values is a non-trivial result. Similarly, the coefficients of $y^{-3}$ and $y^{-5}$ in $D_{1,1,6}$ are linear combinations with rational coefficients of single-valued multiple zeta of weight 11 and 13 respectively [43].

Another important aspect of the structure described in this paper is that the modular graph functions that arise at special values of the single-valued elliptic multiple polylogarithms can be expressed as integrals of sums of single-valued multiple polylogarithms. This means that their functional form can be determined by using the algebraic properties of multiple polylogarithms that were described in section 2. Although this is an interesting comment on the structure of these functions, it is quite complicated to evaluate the functions in this manner, even for the simplest nontrivial 
function which is $C_{1,1,1}(q)$, which is evaluated explicitly in appendix B. The result has previously been obtained by Zagier (unpublished) and in [9] by other methods.

\section{Summary And Thoughts on the Basis of MODUlar GRAPH FUnCTIONS}

The preceding discussion concerns properties of modular graph functions that are associated with Feynman diagrams for a free conformal scalar field on a torus. A general graph, denoted $\Gamma=\Gamma_{w, N}$, has $w$ Green functions joining $N$ vertices at positions $\zeta_{i}(i=1, \ldots, N)$ that are integrated over the torus. Each Green function is itself a single-valued elliptic polylogarithm, $D_{1,1}\left(q, \zeta_{i} / \zeta_{j}\right)$. This procedure defines a modular function, $I_{\Gamma}(q)$, associated with the graph as in (3.10).

We also considered the graph with $N+1$ vertices that is obtained from $\Gamma=\Gamma_{w, N}$ by integrating over only $N-1$ vertices and separating the Green functions joined to the $N$-th vertex into two groups groups that end at the points $\zeta_{N}$ and $\zeta_{N+1}$. This is associated with the function $I_{\Gamma}(q ; \zeta)$ (3.14), which is a single-valued function of the argument $\zeta=\zeta_{N+1} / \zeta_{N}$. This is a particular example of a single-valued elliptic multiple polylogarithm. The value of this function at the point $\zeta=1$ is a modular graph function, $I_{\Gamma}(q ; 1)=I_{\Gamma}(q)$.

As in the case of multiple zeta values the question of determining a basis of modular graph functions seems daunting. In considering this question we should bear in mind that the context that motivated our analysis was limited. The general Feynman diagrams with $N$ vertices and $w$ Green functions that were considered in this paper and in [9-11] generalise those obtained by expanding the four-graviton superstring scattering amplitude, which only generates the diagrams with $N \leq 4$. This does not provide a complete set of basis functions. In fact, the relations between the functions that were proved or conjectured in [9] explicitly involve relations between functions with at most four vertices and those with more than four vertices.

The preceding comments parallel known properties of the expansion of the tree-level $N$-graviton amplitude, which generates a subset of single-valued multi-zeta values at any weight. For example for $N=4$ the expansion coefficients are polynomial in ordinary odd Riemann zeta values. In order to access the complete basis of multiple zeta values it is necessary to expand the tree amplitudes for general $N$ [4,32]. By analogy, we would expect that a more complete understanding of the basis for the space of modular graph functions requires analysis of the low energy expansion of $N$ graviton genus-one amplitudes, which is presently rather limited. It is known [42] that for $N=5$ new kinds of modular graph functions appear for weights $w>4$. These are graphs in which $2 N-8$ Green functions contain a holomorphic or anti-holomorphic numerator momentum factor, which have the form

$$
D_{1,0}(q ; \zeta)=-\zeta \partial_{\zeta} D_{1,1}(q ; \zeta), \quad D_{0,1}(q ; \zeta)=-\bar{\zeta} \partial_{\bar{\zeta}} D_{1,1}(q ; \zeta)
$$

These functions transform as modular forms of non-zero modular weight as seen from (2.35). Such graphs are integrals of products of $D_{a, b}(q ; \zeta)$ with $a, b=0,1$, with the 
constraint that the integrand has zero net modular weight. For example the integrands of the modular graph functions of this type in the $N=5$ case have one $D_{0,1}$ factor and one $D_{1,0}$ factor.

Since the relationships between different modular graph functions are difficult to determine with present techniques, it is not clear how many independent modular weight functions there are at any weight.

The fact that the coefficients of the Laurent polynomials of the constant Fourier mode of modular graph functions are single-valued multiple zeta values suggests that they may be related to coefficients in the low energy expansion of tree-level amplitudes. After all, in the limit $\tau_{2} \rightarrow \infty$ the genus-one $N$-graviton amplitude degenerates to a $N+2$-particle tree amplitude at special values of the momenta. It would therefore be interesting to connect these coefficients to the single-valued multiple zetas that appear in the low energy expansion of tree-level closed string amplitudes $[4,5]$.

A related issue is a possible connection between modular graph functions and holomorphic elliptic multiple polylogarithms of [14]. The latter arise in the low energy expansion of open string theory, as discussed in [15]. Such a connection would be analogous to the relation of ordinary multiple polylogarithms to single-valued multiple polylogarithms. Within string theory this corresponds to the KLT relation that builds the tree level closed string amplitudes from the open string tree amplitudes. There are strong hints that the integrands of the open string one-loop amplitude and the closed string genus-one amplitude considered in this paper are related in an analogous manner. Determining the details of such a genus-one "KLT" relation would be very enlightening.

\section{ACKNOWLEDGMENTS}

We would like thank Erik Panzer and Don Zagier for many useful discussions and exchanges. We are very grateful to Federico Zerbini for providing us with his evaluation of the Laurent polynomials of various graphs prior to publication. We would also like to thank both referees of this paper for their detailed and very helpful comments.

The research of ED was supported in part by grants from the National Science Foundation (NSF) PHY-13-13986 and PHY-16-19926. The research of PV has received funding the ANR grant reference QST 12 BS05 003 01, and the CNRS grants PICS number 6430. The research of OG has received funding by European Research Council (Programme Ideas ERC-2012-AdG 320769 AdS-CFT-solvable) and by the ANR grant StrongInt (BLANC-SIMI-4-2011). PV is partially supported by a fellowship funded by the French Government at Churchill College, Cambridge. MBG and PV acknowledge funding from the European Research Council under the European Community's Seventh Framework Programme (FP7/2007-2013) ERC grant agreement no. [247252]. 


\section{Appendix A. Polylogarithms And Eichler integrals}

We will here demonstrate an interesting connection between elliptic polylogarithms and integrals of holomorphic Eisenstein series. Part of this discussion is contained in $[44, \S 7]$.

To begin with we will introduce a set of derivatives that shows how the $D_{a, b}(q ; \zeta)$ functions of section 2.7 can be expressed as derivatives of the polylogarithms and holomorphic Eisenstein series. Consider the differential operator

$$
\mathbb{D}_{a}=(-1)^{a-1} \sum_{k=0}^{a-1}\left(\begin{array}{c}
2 a-2-k \\
a-1
\end{array}\right) \frac{\left(-\log |x|^{2}\right)^{k}}{k !}\left(\frac{d}{d \log x}\right)^{k} .
$$

Making use of the differentiation relation (2.6) one can express $D_{a, a}(x)$ as

$$
D_{a, a}(x)=2 \Re \mathrm{e}\left(\mathbb{D}_{a} \mathrm{Li}_{2 a-1}(x)\right) .
$$

The action of $\mathbb{D}_{a}$ can be promoted to the action of a covariant derivative

$$
\mathcal{D}_{a}=(-1)^{a-1} \sum_{k=0}^{a-1}\left(\begin{array}{c}
2 a-2-k \\
a-1
\end{array}\right) \frac{\left(4 \pi \tau_{2}\right)^{k}}{k !}\left(\frac{d}{d \log q}\right)^{k}
$$

which maps weight $\ell$ modular forms to weight $k+\ell$ modular forms. This differential operator can be expressed as the product of covariant derivatives [44, $\S 7]$

$$
\mathcal{D}_{a}=\left(4 \pi \tau_{2}\right)^{m-1}\left(\partial_{-2} \circ \partial_{-4} \circ \cdots \circ \partial_{2-2 a}\right)
$$

where $\partial_{k}=\frac{1}{2 i \pi} \partial_{\tau}-\frac{k}{4 \pi \tau_{2}}$. Equipped with $\mathcal{D}_{a}$ one can rewrite (A.2) in the form

$$
D_{a, a}(q ; \zeta)=2 \Re \mathrm{e} \mathcal{D}_{a}\left[\widetilde{G}_{2 a-1}(q ; \zeta)\right]
$$

where

$$
\widetilde{G}_{2 a-1}(q ; \zeta)=\sum_{n \geq 0}\left(\operatorname{Li}_{2 a-1}\left(q^{n} \zeta\right)+\operatorname{Li}_{2 a-1}\left(q^{n+1} / \zeta\right)\right)-\frac{(\log q)^{2 a-1}}{(2 a) !} B_{2 a}(u) .
$$

Setting $\zeta=1$ and using $B_{2 a}(1)=-2 a \zeta(1-2 a)$ leads to an expression for the Eisenstein series as

$$
E_{a}(q)=\frac{1}{\left(-4 \pi \tau_{2}\right)^{a-1}} 2 \Re \mathrm{e} \mathcal{D}_{a}\left[\widetilde{G}_{2 a-1}(q ; 1)\right]
$$

where the function $\widetilde{G}_{2 a-1}(q ; 1)$ is given by

$$
\widetilde{G}_{2 a-1}(q)=\zeta(1-2 a) \frac{(\log q)^{2 a-1}}{(2 a-1) !}+\zeta(2 a-1)+2 \sum_{n=1}^{\infty} \operatorname{Li}_{2 a-1}\left(q^{n}\right) .
$$


In fact, $\widetilde{G}_{2 a-1}(q)$ is an Eichler integral ${ }^{7}$ that satisfies

$$
\left(\frac{d}{d \log q}\right)^{2 a-1} \widetilde{G}_{2 a-1}(q)=2 G_{2 a}(q) .
$$

The quantity $G_{2 a}(q)$ weight $2 a$ holomorphic Eisenstein series for $S L(2, \mathbb{Z})$, defined by

$$
G_{2 a}(q)=\frac{(2 a-1) !}{2(2 i \pi)^{2 a}} \sum_{(m, n) \neq(0,0)} \frac{1}{(m \tau+n)^{2 a}}=\frac{1}{2} \zeta(1-2 a)+\sum_{n=1}^{\infty} n^{2 a-1} \frac{q^{n}}{1-q^{n}} .
$$

Since $\operatorname{Li}_{0}(x)=\frac{x}{1-x}$ the $q$-expansion is given by a weight 0 polylogarithm as implied by the relation (A.9). Combining (A.5) with (A.9) leads to a relation between the nonholomorphic Eisenstein series $E_{a}(q)$ and the holomorphic Eisenstein series, $G_{2 a}(q)$.

At the end of the next appendix we will use a similar argument to obtain relations between both the modular functions $E_{3}(q)$ and $C_{1,1,1}(q)$ and the holomorphic Eisenstein series $G_{6}(q)$.

\section{Appendix B. The $C_{1,1,1}(q)$ modular graph function}

In this section we illustrate how the connection between modular graph functions and single-valued multiple polylogarithms can be used to evaluate their functional form. We will here consider the first nontrivial example, which is the function $C_{1,1,1}(q)$ that is defined by

$$
C_{1,1,1}(q)=\int_{\Sigma} \frac{d^{2} \log \zeta}{4 \pi^{2} \tau_{2}} D_{1,1}(q ; \zeta)^{3}
$$

with $D_{1,1}(q ; \zeta)$ defined in $(3.9)$ and $\zeta=q^{u} e^{2 i \pi v}$ with $u, v \in[0,1]$.

We now expand the integrand using the stuffle relations of polylogarithms obtained using the series representation

$$
\begin{aligned}
\prod_{i=1}^{3} \operatorname{Li}_{a_{i}}\left(x_{i}\right)= & \operatorname{Li}_{a_{1}+a_{2}+a_{3}}\left(x_{1} x_{2} x_{3}\right)+\sum_{\sigma \in \mathfrak{S}_{3}} \operatorname{Li}_{a_{\sigma(1)}, a_{\sigma(2)}, a_{\sigma(3)}}\left(x_{\sigma(1)}, x_{\sigma(2)}, x_{\sigma(3)}\right) \\
& +\sum_{i=1}^{3} \delta_{\{i, j k\}=\{1,2,3\}}\left(\operatorname{Li}_{a_{i}, a_{j}+a_{k}}\left(x_{i}, x_{j} x_{k}\right)+\operatorname{Li}_{a_{j}+a_{k}, a_{i}}\left(x_{j} x_{k}, x_{i}\right)\right),
\end{aligned}
$$

where $x_{i}$ are the arguments of the polylogarithms that are of the form in the various terms of (3.9). The symbol $\mathfrak{S}_{3}$ denotes the set of permutations of three elements, and $\delta_{\{i, j k\}=\{1,2,3\}}$ enforces the constraint that $(i, j, k)$ is a permutation of $(1,2,3)$. We apply this identity with $a_{i}=1,2$

7 There is a polynomial ambiguity $\sum_{i=0}^{2 a-2} a_{i}(\log q)^{i}$ where $a_{i}$ are constants. This polynomial is the period polynomial of the modular form as discussed in [45]. 
As an illustration we evaluate the contribution from the term in the integrand with only the factors of $\mathrm{Li}_{2}$. This has the form

$$
\begin{aligned}
I_{2,2,2} & =\frac{\tau_{2}^{3}}{\pi^{3}} \int_{\Sigma} \frac{d^{2} \log \zeta}{4 \pi^{2} \tau_{2}}\left(\operatorname{Li}_{2}\left(e^{2 i \pi u}\right)+\operatorname{Li}_{2}\left(e^{-2 i \pi u}\right)\right)^{3} \\
& =\frac{6 \tau_{2}^{3}}{\pi^{3}} \Re \mathrm{e} \int_{0}^{1} d u \operatorname{Li}_{2}\left(e^{2 i \pi u}\right)^{2} \operatorname{Li}_{2}\left(e^{-2 i \pi u}\right)
\end{aligned}
$$

Substituting the expansion (B.2)

$$
\begin{aligned}
& \operatorname{Li}_{2}\left(e^{2 i \pi u}\right)^{2} \operatorname{Li}_{2}\left(e^{-2 i \pi u}\right)=\operatorname{Li}_{6}\left(e^{2 i \pi u}\right)+2 \operatorname{Li}_{2,2,2}\left(e^{2 i \pi u}, e^{2 i \pi u}, e^{-2 i \pi u}\right) \\
& +2 \operatorname{Li}_{2,2,2}\left(e^{2 i \pi u}, e^{-2 i \pi u}, e^{2 i \pi u}\right)+2 \operatorname{Li}_{2,2,2}\left(e^{-2 i \pi u}, e^{2 i \pi u}, e^{2 i \pi u}\right) \\
& +2 \operatorname{Li}_{2,4}\left(e^{2 i \pi u}, 1\right)+2 \operatorname{Li}_{4,2}\left(1, e^{2 i \pi u}\right)+\operatorname{Li}_{2,4}\left(e^{-2 i \pi u}, e^{4 i \pi u}\right)+\operatorname{Li}_{4,2}\left(e^{4 i \pi u}, e^{-2 i \pi u}\right)
\end{aligned}
$$

and using the series expression for the multiple polylogarithms we find that the only non vanishing contributions are

$$
\begin{aligned}
I_{2,2,2} & =\frac{6 \tau_{2}^{3}}{\pi^{3}} \Re \mathrm{e} \int_{0}^{1} d u\left(2 \operatorname{Li}_{2,2,2}\left(e^{2 i \pi u}, e^{2 i \pi u}, e^{-2 i \pi u}\right)+\operatorname{Li}_{4,2}\left(e^{4 i \pi u}, e^{-2 i \pi u}\right)\right) \\
& =\frac{6 \tau_{2}^{3}}{\pi^{3}} \Re \mathrm{e}\left(2 \sum_{0<m_{1}<m_{2}} \frac{1}{m_{1}^{2} m_{2}^{2}\left(m_{1}+m_{2}\right)^{2}}+\frac{1}{4} \operatorname{Li}_{6}(1)\right) .
\end{aligned}
$$

The expression in parenthesis is real and is a special value of the multiple sum

$$
L_{a, b ; c}(x, y)=\sum_{m_{1}, m_{2} \geq 1} \frac{x^{m_{1}} y^{m_{2}}}{m_{1}^{a} m_{2}^{b}\left(m_{1}+m_{2}\right)^{c}} .
$$

since

$$
\begin{aligned}
L_{a, b ; c}(x, y)=\frac{1}{2^{c}} \sum_{0<m} \frac{(x y)^{m}}{m^{a+b+c}} & \\
& +\sum_{0<m_{1}<m_{2}} \frac{x^{m_{1}} y^{m_{2}}}{m_{1}^{a} m_{2}^{b}\left(m_{1}+m_{2}\right)^{c}}+\sum_{0<m_{2}<m_{1}} \frac{x^{m_{1}} y^{m_{2}}}{m_{1}^{a} m_{2}^{b}\left(m_{1}+m_{2}\right)^{c}} .
\end{aligned}
$$

The multiple sums $L_{a, b ; c}(x, y)$ may be reduced to a linear combination of multiple polylogarithms as shown in appendix $\mathrm{C}$, with the result

$$
L_{2,2 ; 2}(x, y)=\operatorname{Li}_{2,4}\left(\frac{y}{x}, x\right)+\operatorname{Li}_{2,4}\left(\frac{x}{y}, y\right)+2 \operatorname{Li}_{1,5}\left(\frac{y}{x}, x\right)+2 \operatorname{Li}_{1,5}\left(\frac{x}{y}, y\right),
$$

and the value at $x=y=1$ is easily obtained using Hyperint routines of [24]

$$
L_{2,2 ; 2}(1,1)=2 \operatorname{Li}_{2,4}(1,1)+4 \operatorname{Li}_{1,5}(1,1)=\frac{\zeta(6)}{3} .
$$


Therefore

$$
I_{2,2,2}=2 \zeta(6) \frac{\tau_{2}^{3}}{\pi^{3}}
$$

The remaining integrals are performed similarly. Collecting everything we have

$$
C_{1,1,1}(q)=\frac{2 \zeta(6)}{\pi^{3}} \tau_{2}^{3}+\varphi_{0}(q)+\frac{\varphi_{1}(q)}{\tau_{2}}+\frac{\varphi_{2}(q)}{\tau_{2}^{2}}
$$

where

$$
\varphi_{0}=\zeta(3)+4 \sum_{n \geq 1} \Re \mathrm{eLi}_{3}\left(q^{n}\right)+12 \sum_{n_{1}, n_{2}, n_{3} \geq 1} \Re \mathrm{e} L_{1,1 ; 1}\left(q^{n_{1}+n_{3}+1}, q^{n_{2}+n_{3}+1}\right),
$$

and

$$
\begin{aligned}
\varphi_{1}(q) & =-\frac{3}{\pi} \sum_{n_{1}, n_{2} \geq 0} \Re \mathrm{e}\left(\operatorname{Li}_{4}\left(q^{n_{1}} \bar{q}^{n_{2}}\right)+\operatorname{Li}_{4}\left(q^{n_{1}+1} \bar{q}^{n_{2}+1}\right)\right) \\
& +\frac{3}{\pi} \sum_{n_{1}, n_{2}, n_{3}>0} \Re \mathrm{e}\left(L_{1,1 ; 2}\left(q^{n_{1}} \bar{q}^{n_{3}}, q^{n_{2}} \bar{q}^{n_{3}}\right)-L_{1,1 ; 2}\left(q^{n_{1}+1} \bar{q}^{n_{3}+1}, q^{n_{2}+1} \bar{q}^{n_{3}+1}\right)\right) \\
& +\frac{6}{\pi} \sum_{n_{1}, n_{2}, n_{3} \geq 0} \Re \mathrm{e}\left(L_{2,1 ; 1}\left(q^{n_{1}} \bar{q}^{n_{3}}, \bar{q}^{n_{2}+n_{3}+1}\right)-L_{2,1 ; 1}\left(q^{n_{1}+1} \bar{q}^{n_{3}+1}, \bar{q}^{n_{2}+n_{3}+1}\right)\right)
\end{aligned}
$$

and finally

$$
\varphi_{2}(q)=-\frac{3}{4 \pi^{2}} \zeta(5)+\frac{3}{2 \pi^{2}} \sum_{n \geq 0} \Re \operatorname{Li}_{5}\left(q^{n}\right) .
$$

We note that the Eisenstein series $E_{3}(q)$ has an expansion that follows from (A.7) of the form

$$
\begin{aligned}
E_{3}(q)=\frac{2 \zeta(6)}{\pi^{3}} \tau_{2}^{3}+\frac{3 \zeta(5)}{4 \pi^{2} \tau_{2}^{2}}+\sum_{n \geq 1}\left(2 n^{2} \Re \operatorname{RLi}_{3}\left(q^{n}\right)+\frac{3 n}{\pi \tau_{2}} \Re \mathrm{eii}_{4}\left(q^{n}\right)\right. & \\
& \left.+\frac{3}{2 \pi^{2} \tau_{2}^{2}} \Re \operatorname{Lii}_{5}\left(q^{n}\right)\right) .
\end{aligned}
$$

Putting everything together we have

$$
C_{1,1,1}(q)-E_{3}(q)-\zeta(3)=\Re \mathrm{e}\left(2 \phi_{0}(q)+\frac{3}{\pi} \frac{\phi_{1}(q)}{\tau_{2}}\right)
$$

where

$$
\phi_{0}(q)=6 \sum_{n_{1}, n_{2}, n_{3} \geq 0} L_{1,1 ; 1}\left(q^{n_{1}+n_{3}+1}, q^{n_{2}+n_{3}+1}\right)+\sum_{n \geq 1}\left(1-n^{2}\right) \operatorname{Li}_{3}\left(q^{n}\right) .
$$


and

$$
\begin{aligned}
\phi_{1}(q)= & -\sum_{n \geq 1} n \operatorname{Li}_{4}\left(q^{n}\right)+\sum_{n_{1}, n_{2} \geq 0}\left(\operatorname{Li}_{4}\left(q^{n_{1}} \bar{q}^{n_{2}}\right)+\operatorname{Li}_{4}\left(q^{n_{1}+1} \bar{q}^{n_{2}+1}\right)\right) \\
& -\sum_{n_{1}, n_{2}, n_{3} \geq 0}\left(L_{1,1 ; 2}\left(q^{n_{1}} \bar{q}^{n_{3}}, q^{n_{2}} \bar{q}^{n_{3}}\right)-L_{1,1 ; 2}\left(q^{n_{1}+1} \bar{q}^{n_{3}+1}, q^{n_{2}+1} \bar{q}^{n_{3}+1}\right)\right) \\
& -2 \sum_{n_{1}, n_{2}, n_{3} \geq 0}\left(L_{2,1 ; 1}\left(q^{n_{1}} \bar{q}^{n_{3}}, \bar{q}^{n_{2}+n_{3}+1}\right)-L_{2,1 ; 1}\left(q^{n_{1}+1} \bar{q}^{n_{3}+1}, \bar{q}^{n_{2}+n_{3}+1}\right)\right) .
\end{aligned}
$$

It is striking that these identities are not reducible to standard polylogarithm identities and that they mix the $q$ and $\bar{q}$ expansion as is seen in the expression for $\phi_{1}(q)$.

Using the expressions for $L_{a, b ; c}(x, y)$ derived in appendix $\mathrm{C}$

$$
\begin{aligned}
& L_{1,1 ; 1}(x, y)=\operatorname{Li}_{1,2}(y / x, x)+\operatorname{Li}_{1,2}(x / y, y) \\
& L_{1,1 ; 2}(x, y)=\operatorname{Li}_{1,3}(y / x, x)+\operatorname{Li}_{1,3}(x / y, y) \\
& L_{2,1 ; 1}(x, y)=\operatorname{Li}_{2,2}(x / y, y)+\operatorname{Li}_{1,3}(y / x, x)+\operatorname{Li}_{1,3}(x / y, y) .
\end{aligned}
$$

one can easily check that $\phi_{0}(q)=0=\phi_{1}(q)$ to an arbitrary order in the $q$ expansion using HyperInt [24]. It then follows from (B.16) that

$$
C_{1,1,1}(q)=E_{3}(q)+\zeta(3) .
$$

B.1. Remark on Eichler integrals. Introducing the covariant derivative

$$
\mathcal{D}_{2}=-2-4 \pi \tau_{2} \frac{d}{d \log q}
$$

we find that

$$
E_{3}(q)=-\frac{1}{\left(-4 \pi \tau_{2}\right)^{2}} 2 \Re \mathrm{e} \mathcal{D}_{2} \widetilde{G}_{5}(q)
$$

and

$$
C_{1,1,1}(q)=-\frac{1}{\left(-4 \pi \tau_{2}\right)^{2}} 2 \Re \mathrm{e} \mathcal{D}_{2} \widehat{G}_{5}(q),
$$

where

$$
\widetilde{G}_{5}(q)=\zeta(-5) \frac{(\log q)^{6}}{5 !}+\zeta(5)+2 \sum_{n=1}^{\infty} \operatorname{Li}_{5}\left(q^{n}\right)
$$

and

$$
\widehat{G}_{5}(q)=\widetilde{G}_{5}(q)+\frac{1}{2} \pi^{3} \zeta(3)(\log q)^{2} .
$$

Both of these function satisfy

$$
\left(\frac{d}{d \log q}\right)^{5} \widetilde{G}_{5}(q)=\left(\frac{d}{d \log q}\right)^{5} \widehat{G}_{5}(q)=2 G_{6}(q) .
$$


where $G_{6}(q)$ is the holomorphic Eisenstein series

$$
G_{6}(q)=\frac{60}{(2 i \pi)^{6}} \sum_{(m, n) \neq(0,0)} \frac{1}{(m \tau+n)^{6}} .
$$

This means that $E_{3}(q)$ and $C_{1,1,1}(q)$ are related to two Eichler integrals of the holomorphic weight 6 Eisenstein series for $S L(2, \mathbb{Z})$. The difference between $\widetilde{G}_{5}(q)$ and $\widehat{G}_{5}(q)$ is the polynomial ambiguity related to the period polynomial arising when integrating the holomorphic Eisenstein series. We refer back to the appendix A for a review of this construction.

\section{Appendix C. Reduction of multiple sums to multiple polylogarithms}

In this appendix we wil reduce various multiple constrained sums to multiple polylogarithms, making use of the partial fractions identity given in [20]

$$
\frac{1}{m^{a} n^{b}}=\sum_{r=b}^{a+b-1} \frac{\left(\begin{array}{l}
r-1 \\
b-1
\end{array}\right)}{(m+n)^{r} m^{a+b-r}}+\sum_{r=a}^{a+b-1} \frac{\left(\begin{array}{l}
r-1 \\
a-1
\end{array}\right)}{(m+n)^{r} n^{a+b-r}} .
$$

These reductions have been checked with the program HyperInt [24]. ${ }^{8}$

We will now reduce the multiple constrained sum $L_{a, b ; c}(x, y)$ that arose in the evaluation of $C_{1,1,1}(q)$ to a sum of multiple polylogarithms, which is given by

$$
L_{a, b ; c}(x, y)=\sum_{m_{1}, m_{2} \geq 1} \frac{x^{m_{1}} y^{m_{2}}}{m_{1}^{a} m_{2}^{b}\left(m_{1}+m_{2}\right)^{c}}
$$

can be expressed, when $a, b>0$, in the form

$$
L_{a, b ; c}(x, y)=\sum_{\substack{r+s=a+b \\
r, s>0}}\left(\left(\begin{array}{l}
r-1 \\
a-1
\end{array}\right) \operatorname{Li}_{s, c+r}(y / x, x)+\left(\begin{array}{l}
r-1 \\
b-1
\end{array}\right) \operatorname{Li}_{s, c+r}(x / y, y)\right) .
$$

Clearly $L_{a, b ; c}(x, y)=L_{b, a ; c}(y, x)$ and

$$
\begin{aligned}
& L_{0, b ; c}(x, y)=\operatorname{Li}_{b, c}(y / x, x) \\
& L_{a, b ; 0}(x, y)=\operatorname{Li}_{a}(x) \operatorname{Li}_{b}(y) .
\end{aligned}
$$

One can integrate the differential equation

$$
\left(x \frac{d}{d x}+y \frac{d}{d y}\right)^{c} L_{a, b ; c}(x, y)=\operatorname{Li}_{a}(x) \operatorname{Li}_{b}(y) .
$$

to give

$$
L_{a, b ; c}(x, y)=\frac{1}{(c-1) !} \int_{0}^{\infty} d \alpha \alpha^{c-1} \operatorname{Li}_{a}\left(x e^{-\alpha}\right) \operatorname{Li}_{b}\left(y e^{-\alpha}\right)
$$

\footnotetext{
${ }^{8}$ We thank Erik Panzer for help in performing theses checks.
} 
This integral representation can then be efficiently integrated with HyperInt [24]. The expression can be written in different ways as a consequence of the shuffle algebra. For example

$$
L_{1,1 ; 1}(x, y)=\operatorname{Li}_{1,2}\left(\frac{y}{x}, x\right)+\operatorname{Li}_{1,2}\left(\frac{x}{y}, y\right),
$$

which is easily checked with HyperInt to be equal to

$$
L_{1,1 ; 1}(x, y)=\operatorname{Li}_{3}(x)+\left(\operatorname{Li}_{2}(x)-\operatorname{Li}_{2}\left(\frac{x}{y}\right)\right) \operatorname{Li}_{1}(y)+\operatorname{Li}_{1,2}\left(y, \frac{x}{y}\right) .
$$

Equating these two expressions evaluated at $x=y=1$ implies Euler's famous relation $\zeta(1,2)=\zeta(3)$.

\section{REFERENCES}

[1] M. B. Green and J. H. Schwarz, "Supersymmetrical String Theories," Phys. Lett. B 109, 444 (1982). doi:10.1016/0370-2693(82)91110-8

[2] E. D'Hoker and D. H. Phong, "Two-loop superstrings VI: Non-renormalization theorems and the 4-point function," Nucl. Phys. B 715, 3 (2005) [hep-th/0501197].

[3] H. Gómez and C. R. Mafra, "The Closed-String 3-Loop Amplitude and S-Duality," JHEP 1310 (2013) 217 [arXiv:1308.6567 [hep-th]].

[4] S. Stieberger, "Closed superstring amplitudes, single-valued multiple zeta values and the Deligne associator," J. Phys. A 47 (2014) 155401 [arXiv:1310.3259 [hep-th]].

[5] S. Stieberger and T. R. Taylor, "Closed String Amplitudes as Single-Valued Open String Amplitudes," Nucl. Phys. B 881 (2014) 269 [arXiv:1401.1218 [hep-th]].

[6] F. Brown, "Polylogarithmes multiples uniformes en une variable", C. R. Acad. Sci. Paris, Ser. I 338 (2004) 527-532.

[7] O. Schnetz, "Graphical Functions and Single-Valued Multiple Polylogarithms," Commun. Num. Theor. Phys. 08 (2014) 589 [arXiv:1302.6445 [math.NT]].

[8] F. Brown, "Single-valued Motivic Periods and Multiple Zeta Values," SIGMA 2 (2014) e25 doi:10.1017/fms.2014.18 [arXiv:1309.5309 [math.NT]].

[9] E. D'Hoker, M. B. Green and P. Vanhove, "On the Modular Structure of the Genus-One Type II Superstring Low Energy Expansion," JHEP 1508 (2015) 041 doi:10.1007/JHEP08(2015)041 [arXiv:1502.06698 [hep-th]].

[10] E. D'Hoker, M. B. Green and P. Vanhove, "Proof of a Modular Relation Between 1-, 2- and 3-Loop Feynman Diagrams on a Torus," arXiv:1509.00363 [hep-th].

[11] A. Basu, "Poisson Equation for the Mercedes Diagram in String Theory at Genus One," Class. Quant. Grav. 33 (2016) no.5, 055005 doi:10.1088/0264-9381/33/5/055005 [arXiv:1511.07455 [hepth]].

[12] E. D'Hoker and M. B. Green, "Zhang-Kawazumi Invariants and Superstring Amplitudes," Journal of Number Theory, Vol 144 (2014) page 111 [arXiv:1308.4597 [hep-th]].

[13] E. D'Hoker, M. B. Green, B. Pioline and R. Russo, "Matching the $D^{6} R^{4}$ interaction at two-loops," JHEP 1501, 031 (2015) doi:10.1007/JHEP01(2015)031 [arXiv:1405.6226 [hep-th]].

[14] F. Brown and A. Levin, "Multiple Elliptic Polylogarithms", arXiv:1110.6917

[15] J. Broedel, C. R. Mafra, N. Matthes and O. Schlotterer, "Elliptic Multiple Zeta Values and One-Loop Superstring Amplitudes," JHEP 1507 (2015) 112 doi:10.1007/JHEP07(2015)112 [arXiv:1412.5535 [hep-th]]. 
[16] J. Broedel, N. Matthes and O. Schlotterer, "Relations Between Elliptic Multiple Zeta Values and a Special Derivation Algebra," J. Phys. A 49 (2016) no.15, 155203 doi:10.1088/17518113/49/15/155203 [arXiv:1507.02254 [hep-th]].

[17] M. B. Green and P. Vanhove, "The Low-energy expansion of the one loop type II superstring amplitude," Phys. Rev. D 61 (2000) 104011 [hep-th/9910056].

[18] M. B. Green, J. G. Russo and P. Vanhove, "Low energy expansion of the four-particle genus-one amplitude in type II superstring theory," JHEP 0802 (2008) 020 [arXiv:0801.0322 [hep-th]].

[19] M. Waldschmidt, "Multiple Polylogarithms: An Introduction." A. K. Agarwal, Bruce C. Berndt, Christian F. Krattenthaler, Gary L. Mullen, K. Ramachandra and Michel Waldschmidt. Conference on number theory and discrete mathematics in honour of Srinivasa Ramanujan, Oct 2000, Chandigarh, India. Hindustan Book Agency. [hal-00416166]

[20] D. Zagier "The Bloch-Wigner-Ramakrishnan polylogarithm function", Math. Ann. 286 (1990), no. $1-3,613-624$.

[21] A. B. Goncharov, "Multiple polylogarithms, cyclotomy and modular complexes", Math.Res.Lett. 5 (1998) 497-516. arXiv:1105.2076,

[22] J. M. Borwein, D. M. Bradley, D. J. Broadhurst, P. Lisoněk, "Special values of multiple polylogarithms," Trans. Amer. Math. Soc. 353 (3) (2001) 907-941. arXiv:math/9910045

[23] K. T. Chen, "Iterated Integrals of Differential Forms and Loop Space Homology", The Annals of Mathematics 97 (2) (1973) 217-246.

[24] E. Panzer, "Algorithms for the Symbolic Integration of Hyperlogarithms with Applications to Feynman Integrals," Comput. Phys. Commun. 188 (2014) 148 [arXiv:1403.3385 [hep-th]].

[25] C. Bogner, "Mpl-A Program for Computations with Iterated Integrals on Moduli Spaces of Curves of Genus Zero," Comput. Phys. Commun. 203 (2016) 339 doi:10.1016/j.cpc.2016.02.033 [arXiv:1510.04562 [physics.comp-ph]].

[26] S. Moch, P. Uwer and S. Weinzierl, "Nested Sums, Expansion of Transcendental Functions and Multiscale Multiloop Integrals," J. Math. Phys. 43 (2002) 3363 doi:10.1063/1.1471366 [hep$\mathrm{ph} / 0110083]$.

[27] S. Weinzierl, "Symbolic Expansion of Transcendental Functions," Comput. Phys. Commun. 145 (2002) 357 doi:10.1016/S0010-4655(02)00261-8 [math-ph/0201011].

[28] S. Moch and P. Uwer, "Xsummer: Transcendental Functions and Symbolic Summation in Form," Comput. Phys. Commun. 174 (2006) 759 doi:10.1016/j.cpc.2005.12.014 [math-ph/0508008].

[29] V. Del Duca, S. Druc, J. Drummond, C. Duhr, F. Dulat, R. Marzucca, G. Papathanasiou and B. Verbeek, "Multi-Regge Kinematics and the Moduli Space of Riemann Spheres with Marked Points," JHEP 1608 (2016) 152 doi:10.1007/JHEP08(2016)152 [arXiv:1606.08807 [hep-th]].

[30] D. Zagier, "Values of zeta functions and their application." First European Congress of Mathematics (Paris, 1992), Vol. II Progr. Math. 120, Birkhäuser 497-512, 1994.

[31] F. C. S. Brown, "Multiple Zeta Values and Periods of Moduli Spaces $\mathfrak{M}_{0, N}(\mathbb{R})$," Annales Sci. Ecole Norm. Sup. 42 (2009) 371 [arXiv:math/0606419 [math.AG]].

[32] O. Schlotterer and S. Stieberger, "Motivic Multiple Zeta Values and Superstring Amplitudes," J. Phys. A 46 (2013) 475401 doi:10.1088/1751-8113/46/47/475401 [arXiv:1205.1516 [hep-th]].

[33] J. Broedel, O. Schlotterer, S. Stieberger and T. Terasoma, "All Order $\alpha^{\prime}$-expansion of Superstring Trees from the Drinfeld Associator," Phys. Rev. D 89 (2014) 6, 066014 doi:10.1103/PhysRevD.89.066014 [arXiv:1304.7304 [hep-th]].

[34] J. Broedel, O. Schlotterer and S. Stieberger, "Polylogarithms, Multiple Zeta Values and Superstring Amplitudes," Fortsch. Phys. 61 (2013) 812 doi:10.1002/prop.201300019 [arXiv:1304.7267 [hep-th]]. 
[35] D. J. Broadhurst and D. Kreimer, "Association of Multiple Zeta Values with Positive Knots via Feynman Diagrams Up to 9 Loops," Phys. Lett. B 393 (1997) 403 doi:10.1016/S03702693(96)01623-1 [hep-th/9609128].

[36] J. Blumlein, D. J. Broadhurst and J. A. M. Vermaseren, "The Multiple Zeta Value Data Mine," Comput. Phys. Commun. 181 (2010) 582 doi:10.1016/j.cpc.2009.11.007 [arXiv:0907.2557 [math$\mathrm{ph}]$.

[37] O. Schnetz: Maple package: Zeta procedures (private communication).

[38] S. Bloch and P. Vanhove, "The Elliptic Dilogarithm for the Sunset Graph," J. Number Theory 148, 328-364 (2015). doi:10.1016/j.jnt.2014.09.032 [arXiv:1309.5865 [hep-th]].

[39] L. Adams, C. Bogner and S. Weinzierl, "The Two-Loop Sunrise Graph in Two Space-Time Dimensions with Arbitrary Masses in Terms of Elliptic Dilogarithms," J. Math. Phys. 55 (2014) 10, 102301 doi:10.1063/1.4896563 [arXiv:1405.5640 [hep-ph]].

[40] L. Adams, C. Bogner and S. Weinzierl, "The Iterated Structure of the All-Order Result for the Two-Loop Sunrise Integral," J. Math. Phys. 57 (2016) no.3, 032304 doi:10.1063/1.4944722 [arXiv:1512.05630 [hep-ph]].

[41] S. Bloch, M. Kerr and P. Vanhove, "A Feynman Integral via Higher Normal Functions," Compositio Mathematica, doi:10.1112/S0010437X15007472 arXiv:1406.2664 [hep-th].

[42] M. B. Green, C. R. Mafra and O. Schlotterer, "Multiparticle one-loop amplitudes and Sduality in closed superstring theory," JHEP 1310 (2013) 188 doi:10.1007/JHEP10(2013)188 [arXiv:1307.3534 [hep-th]].

[43] F. Zerbini, "Single-Valued Multiple Zeta Values in Genus 1 Superstring Amplitudes," arXiv:1512.05689 [hep-th].

[44] D. Zagier and H. Gangl, "Classical and elliptic polylogarithms and special values of L-series," The arithmetic and geometry of algebraic cycles (Banff, AB, 1998), NATO Sci. Ser. C Math. Phys. Sci. 548, Kluwer Acad. Publ., Dordrecht 2000, 561-615.

[45] D. Zagier, "Periods of modular forms and Jacobi theta functions", Invent. Math. 104 (1991), 449-465

Eric D'Hoker, Mani L. Bhaumik Institute for Theoretical Physics, Department of

Physics and Astronomy, University of California, Los Angeles, CA 90095, USA

E-mail address: dhoker@physics.ucla.edu

Michael B. Green, Department of Applied Mathematics and Theoretical Physics, Wilberforce Road, Cambridge CB3 0WA, UK

E-mail address: M.B.Green@damtp.cam.ac.uk

Ömer GürdoĞan, Institut de Physique Théorique, CEA, IPhT, F-91191 Gif-Sur-Yvette, France, CNRS, URA 2306, F-91191 Gif-Sur-Yvette, France

Laboratoire de Physique Théorique de l'École Normale Supérieure, 24, Rue Lhomond, 75231 Paris CEDEX, France

E-mail address: omer.gurdogan@cea.fr

Pierre Vanhove, Institut des Hautes Etudes Scientifiques, Le Bois-Marie, 35 route de Chartres, F-91440 Bures-Sur-Yvette, France

Institut de Physique Théorique, CeA, IPht, F-91191 Gif-sur-Yvette, France, CNRS, URA 2306, F-91191 Gif-Sur-Yvette, France

Department of Applied Mathematics and Theoretical Physics, Wilberforce Road, CAmbridge CB3 0WA, UK

E-mail address: pierre.vanhove@cea.fr 